$\xi=$

\title{
Comparison of estimates using censored samples from Gompertz model: Bayesian, E-Bayesian, hierarchical Bayesian and empirical Bayesian schemes
}

\author{
Hesham Mohamed Reyad ${ }^{1,2 *}$, Adil Mousa Younis ${ }^{1,2}$, Amal Alsir Alkhedir ${ }^{2}$ \\ ${ }^{1}$ College of Business and Economics, Qassim University, Kingdom of Saudi Arabia \\ ${ }^{2}$ Faculty of Science, Sudan University of Science and Technology, Sudan \\ *Corresponding author E-mail: hesham_reyad@yahoo.com
}

\begin{abstract}
This paper aims to introduce a comparative study for the E-Bayesian criteria with three various Bayesian approaches; Bayesian, hierarchical Bayesian and empirical Bayesian. This study is concerned to estimate the shape parameter and the hazard function of the Gompertz distribution based on type-II censoring. All estimators are obtained under symmetric loss function [squared error loss (SELF))] and three different asymmetric loss functions [quadratic loss function (QLF), entropy loss function (ELF) and LINEX loss function (LLF)]. Comparisons among all estimators are achieved in terms of mean square error (MSE) via Monte Carlo simulation.
\end{abstract}

Keywords: Bayes estimates; E-Bayes estimates; Empirical Bayes estimates; Gompertz distribution; Hierarchical Bayes estimates.

\section{Introduction}

The Gompertz distribution has great importance in modeling human mortality and actuarial tables. It has many applications, particularly in medical and actuarial studies. Also, it used as a survival model in reliability. Historically, the Gompertz distribution was first proposed by Gompertz [1]. The probability density function (pdf), cumulative distribution function (cdf), and hazard function $h(t)$ of the two-parameter Gompertz distribution are given, respectively, by

$$
\begin{array}{ll}
f(x ; \lambda, \theta)=\lambda \theta \exp \left[\lambda x-\theta\left(e^{\lambda x}-1\right)\right], & x>0, \lambda, \theta>0, \\
F(x ; \lambda, \theta)=1-\exp \left[-\theta\left(e^{\lambda x}-1\right)\right], & x>0, \lambda, \theta>0
\end{array}
$$

And

$$
h(t ; \lambda, \theta)=\lambda \theta \exp (\lambda t), \quad t>0, \lambda, \theta>0
$$

Where $\lambda$ and $\theta$ are the scale and shape parameters respectively. Recently, many authors have studied the Gompertz distribution; for example, Grag [2] discussed the properties of the Gompertz distribution and estimate its parameters by using the maximum likelihood method. Chen [3] reproduced an exact confidence interval and exact joint confidence region for the parameters associated to the Gompertz distribution based on type-II censoring. Jaheen [4] constructed the Bayesian technique for the Gompertz distribution under record values. Wu et al [5] obtained the point and interval estimators for the unknown parameters corresponding to the Gompertz distribution based on progressive type-II censored samples. Gohary [6] introduced the bivariate Gompertz distribu- tion and completed the analysis for the mixture of components of the proposed distribution. Saracoglu et al [7] compared the nonBayes and Bayes estimates for the unknown parameters of the Gompertz distribution. Ismail [8] derived point and interval estimates for the Gompertz distribution based on partially accelerated life tests with type-II censoring. Feroze and Aslam [9] obtained point and interval estimates for the parameters of the twocomponent mixture of the Gompertz model based on Bayes Method along with posterior predictions for the future value from model. Sarabia et al [10] exploded several properties of the Gompertz distribution when lifetime or other kinds of data available fully observed.

The E-Bayesian estimation is a new method of estimation first introduced by Han [11]. Han [12] derived the E-Bayes and hierarchical Bayes estimates of the reliability parameter for testing data from products with exponential distribution under type-I censoring and by considering the quadratic loss function. He proved that via simulation, the E-Bayesian estimator is efficient and easy to operate. Han [13] obtained the E-Bayesian estimation of the failure probability based on type-I censored data and by using the quadratic loss function. Yin and Liu [14] applied the E-Bayesian estimation and hierarchical Bayesian estimation methods for estimating the unknown reliability parameter of the geometric distribution under scaled squared loss function in complete samples. They deducted that the E-Bayes criteria is more stability and convenient in terms of calculation complexity than the hierarchical Bayes method. Han [15] obtained the E-Bayes and hierarchical Bayes estimates of reliability for testing data from products with binomial distribution under type-I censoring and by considering the quadratic loss function. He showed that by using simulation the EBayes technique is much simpler than the hierarchical Bayes method to operate. Wei et al [16] constructed the minimum risk equivariant estimation and E-Bayes estimation methods for estimating the unknown parameter of the Burr-XII distribution based on entropy loss function in complete samples. They deducted that 
E-Bayes estimates have most accuracy. Jaheen and Okasha [17] compared the Bayesian and E-Bayesian estimators for the parameters and reliability function of the Burr Burr-XII distribution based on type-II censoring and by considering the squared error loss and LINEX loss functions. They deducted that the overall performance of the E-Bayes estimates are better than the similar obtained by using the Bayes technique. Cai et al [18] applied the E-Bayesian estimation method for forecasting of security investment. Okasha [19] constructed the maximum likelihood, Bayesian and EBayesian methods for estimating the unknown scale parameter and reliability and hazard functions of the Weibull distribution under type- 2 censored samples and by considering the squared error loss function. He concluded that the E-Bayes estimates were more efficient than the maximum likelihood estimates or the Bayes estimates. $\mathrm{Wu}$ [20] introduced the Bayesian estimation and EBayesian estimation techniques in a new integral interval for estimating the failure probability under zero-failure data and by considering the quadratic loss function. Azimi et al [21] estimated the parameter and reliability function of the generalized half Logistic distribution by using the Bayes and E-Bayes methods based on progressively type-II censoring and by considering the squared error loss and LINEX loss functions. They deducted that the EBayes criteria generally is more efficient than the Bayes criteria. Javadkani et al [22] applied the Bayes, empirical Bayes and EBayes techniques for estimating the unknown shape parameter and the reliability function of the two parameter bathtub-shaped lifetime distribution based on progressively first-failure-censored samples and by considering the minimum expected loss and LINEX loss functions. Liu et al [23] used the E-Bayes and hierarchical methods for estimating the unknown parameter of the Rayleigh distribution under q-symmetric entropy loss function in complete samples. They deducted that the two techniques were close to each other when the sample size is large enough and the E-Bayes estimation was more convenient in terms of calculation complexity. Okasha [24] constructed the Bayesian and the EBayesian methods for estimating the scale parameter, reliability and hazard functions of the Lomax distribution based on type- 2 censored and by considering the balanced squared error loss function. He pointed out that the performance of the E-Bayes estimates is generally better than the Bayes estimates. Reyad and Othman [25] obtained the Bayesian and E-Bayesian estimates for the shape parameter of the Gumbell type-II distribution based on type-II censoring and by considering squared error, LINEX, Degroot, Quadratic and minimum expected loss functions. They deducted that the E-Bayes estimates were generally much better than the other estimates.

The goal of this paper is to introduce a statistical comparison between the E-Bayesian criteria versus other three techniques of Bayesian approaches; Bayesian, hierarchical and empirical Bayesian to illustrate the potential usefulness of the E-Bayesian estimates which are simple in calculations and efficient. The resulting estimates are obtained based on symmetric and different asymmetric loss functions and the all outcomes obtained in this article can be generalized to use in complete sample.

The layout of the paper is as follow. In Section 2, the Bayes estimates of the parameter $\theta$ and the hazard function $h(t)$ based on type-II censored sample are derived under SELF, QLF, ELF and LLF. The E- Bayes estimates are obtained of the parameter $\theta$ and the hazard function $h(t)$ based on type-II censored sample under SELF, QLF, ELF and LLF in Section 3. In Sections 4, 5, the hierarchical Bayes estimates and empirical Bayes of the parameter $\theta$ and the hazard function $h(t)$ are derived based on type-II censored sample under SELF, QLF, ELF and LLF respectively. In Section 6, a Monte Carlo simulation is done to compare the behavior of the resulting estimators. Some concluding remarks have been given in the last Section.

\section{Bayesian estimation}

In this section, we will obtain the Bayes estimates of the shape parameter $\theta$ and the hazard function $h(t)$ of the Gompertz distribution by considering symmetric loss function (SELF)) and three asymmetric loss functions (QLF, ELF and LLF). Based on type-II censored samples of size $r$ obtained from a life test of $n$ items from the Gompertz in (1-1) and (1-2) distribution, the likelihood function can be written as

$$
\begin{aligned}
L(\theta \mid \underline{x}) & =\frac{n !}{(n-r) !} \prod_{i=1}^{r} \lambda \theta \exp \left[\lambda x_{(i)}-\theta\left(e^{\lambda x_{(i)}}-1\right)\right]\left[\exp \left(-\theta\left(e^{\lambda x_{(r)}}-1\right)\right)\right]^{n-r} \\
& \propto \theta^{r} \exp [-\theta Q]
\end{aligned}
$$

Where

$$
Q=\left\{\exp \left[\lambda \sum_{i=1}^{r} x_{(i)}\right]+(n-r)\left[\exp \left(\lambda x_{(r)}\right)\right]-r\right\}
$$

Assuming $\lambda$ is known, we can use the gamma distribution as an conjugate prior distribution of $\theta$ with shape and scale parameter $a$ and $b$ respectively and its pdf given by

$$
g(\theta \mid a, b)=\frac{b^{a}}{\Gamma(a)} \theta^{a-1} \exp [-b \theta], \quad \quad \theta>0, \quad a, b>0
$$

Combining (2-1) and (2-3), from Bayesian theorem the posterior density function of $\theta$ can be obtained as

$$
\begin{aligned}
\pi(\theta \mid \underline{x}) & =\frac{L(\theta \mid \underline{x}) g(\theta \mid a, b)}{\int_{0}^{\infty} L(\theta \mid \underline{x}) g(\theta \mid a, b) d \theta} \\
& =\frac{(Q+b)^{r+a}}{\Gamma(r+a)} \theta^{r+a-1} e^{-(Q+b)}, \quad \theta>0
\end{aligned}
$$

That mean, the posterior distribution of $\theta$ obeys $\Gamma(r+a, Q+b)$.

\subsection{Bayesian estimation under squared error loss func- tion (SELF)}

A commonly used loss function is the square error loss function (SELF) introduced by Mood et al (26) as follows:

$$
L_{1}(\hat{\theta}, \theta)=k(\hat{\theta}-\theta)^{2}, \quad k>0
$$

Where $\hat{\theta}$ is an estimator of $\theta$ and $k$ is the scale of the loss function. The scale $k$ is often taken equal to one which has no effect on the Bayes estimates. This loss function is symmetric in nature. i.e. it gives equal importance to both over and under estimation. The Bayes estimator of $\theta$ denoted by $\hat{\theta}_{B S}$ can be obtained as

$$
\hat{\theta}_{B S}=E_{\pi}(\theta \mid \underline{x})
$$

Where $E_{\pi}$ indicated to the expectation of the posterior distribution. We can derived $\hat{\theta}_{B S}$ by using (2-4) in (2-6) to be

$$
\hat{\theta}_{B S}=\frac{r+a}{Q+b}
$$


We can also obtain the Bayes estimator of $h(t)$ based on SELF denoted as $\hat{h}_{B S}$ by replacing $\hat{\theta}_{B S}$ given in (2-7) instead of $\theta$ given in $(1-3)$ to be

$$
\hat{h}_{B S}=\lambda\left(\frac{r+a}{Q+b}\right) e^{\lambda t}
$$

\subsection{Bayesian estimation under quadratic loss function (QLF)}

Bhuiyan et al [27] defined the quadratic loss function (QLF) as follows:

$$
L_{2}(\hat{\theta}, \theta)=\left(\frac{\theta-\hat{\theta}}{\theta}\right)
$$

The Bayes estimator of $\theta$ based on QLF denoted by $\hat{\theta}_{B Q}$ can be obtained as

$$
\hat{\theta}_{B Q}=\frac{E_{\pi}\left(\theta^{-1} \mid \underline{x}\right)}{E_{\pi}\left(\theta^{-2} \mid \underline{x}\right)}
$$

We can derived $\hat{\theta}_{B Q}$ by using (2-4) in (2-10) to be

$$
\hat{\theta}_{B Q}=\frac{r+a-2}{Q+b}
$$

We can also obtain the Bayes estimator of $h(t)$ based on SELF denoted as $\hat{h}_{B Q}$ by replacing $\hat{\theta}_{B Q}$ given in (2-11) instead of $\theta$ given in (1-3) to be

$$
\hat{h}_{B Q}=\lambda\left(\frac{r+a-2}{Q+b}\right) e^{\lambda t}
$$

\subsection{Bayesian estimation under entropy loss function (ELF)}

Day et al [28] have discussed the entropy loss function (ELF) of the form

$$
L_{3}(\hat{\theta}, \theta) \propto\left(\frac{\hat{\theta}}{\theta}\right)-\ln \left(\frac{\hat{\theta}}{\theta}\right)-1
$$

The Bayes estimator of $\theta$ relative to ELF denoted by $\hat{\theta}_{B E}$ can be obtained as

$$
\hat{\theta}_{B E}=\left[E_{\pi}\left(\theta^{-1} \mid \underline{x}\right)\right]^{-1}
$$

We can obtain $\hat{\theta}_{B E}$ by using (2-4) in (2-14) to be

$$
\hat{\theta}_{B E}=\frac{r+a-1}{Q+b}
$$

The Bayes estimator of $h(t)$ relative to ELF denoted as $\hat{h}_{B E}$ by replacing $\hat{\theta}_{B E}$ given in (2-15) instead of $\theta$ given in (1-3) to be

$$
\hat{h}_{B E}=\lambda\left(\frac{r+a-1}{Q+b}\right) e^{\lambda t}
$$

\subsection{Bayesian estimation under LINEX loss function (LLF)}

Zellner [29] represent the LINEX (linear-exponential) loss function (LLF) to be

$$
L_{4}(\hat{\theta}, \theta)=m\{\exp [s(\hat{\theta}-\theta)]-s(\hat{\theta}-\theta)-1\}
$$

With two parameters $m>0, s \neq 0$, where $m$ is the scale of the loss function and $s$ determines its shape. Without loss of generality, we assume $m=1$. The Bayes estimator relative to LLF denoted by $\hat{\theta}_{B L}$ can be obtained as

$\hat{\theta}_{B L}=\left(\frac{-1}{s}\right) \ln \left[E_{\theta}\left(e^{-s \theta} \mid \underline{x}\right)\right]$

We can obtain $\hat{\theta}_{B L}$ by using (2-4) in (2-18) to be

$\hat{\theta}_{B L}=\left(\frac{r+a}{s}\right) \ln \left[1+\frac{s}{Q+b}\right]$

The Bayes estimator of $h(t)$ relative to LLF denoted as $\hat{h}_{B L}$ by replacing $\hat{\theta}_{B L}$ given in (2-19) instead of $\theta$ given in (1-3) to be

$$
\hat{h}_{B L}=\lambda\left(\frac{r+a}{s}\right) \ln \left[1+\frac{s}{Q+b}\right] e^{\lambda t}
$$

\section{E-Bayesian estimation}

In this section, we will derive the E-Bayes estimates of the shape parameter $\theta$ and the hazard function $h(t)$ of the Gompertz distribution based on symmetric loss function (SELF)) and three asymmetric loss functions (QLF, ELF and LLF). Based on Han [30], the prior parameters $a$ and $b$ must be choose to guarantee that $g(\theta \mid a, b)$ given in (2-3) is a decreasing function of $\theta$. The derivative of $g(\theta \mid a, b)$ with respect to $\theta$ is

$$
\frac{d g(\theta \mid a, b)}{d \theta}=\frac{b^{a}}{\Gamma(a)} \theta^{a-2}[\exp [-b \theta]][(a-1)-b \theta]
$$

Note that $a>0, b>0$ and $\theta>0$ leads to $0<a<1, b>0$ due to $\frac{d g(\theta \mid a, b)}{d \theta}<0$, and therefore $g(\theta \mid a, b)$ is a decreasing function of $\theta$. Suppose that $a$ and $b$ are independent with bivariate density function

$\pi(a, b)=\pi_{1}(a) \pi_{2}(b)$

Then, the E-Bayesian estimate of $\theta$ (expectation of the Bayesian estimate of $\theta$ ) can be written as

$$
\hat{\theta}_{E B}=E(\theta \mid \underline{x})=\iint_{\Omega} \hat{\theta}_{B}(a, b) \pi(a, b) d a d b
$$

Where $\hat{\theta}_{B}(a, b)$ is the Bayes estimate $\theta$ of given by (2-7), (2-11), (2-15) and (2-19). For more details see Han [11, 31]. 


\subsection{E-Bayesian estimation under squared error loss function (SELF)}

E-Bayesian estimates of $\theta$ are derived depending on three different distributions of the hyper-parameters $a$ and $b$. These distributions are used to study the impact of the different prior distributions on the E-Bayesian estimation of $\theta$. The following distributions of $a$ and $b$ may be used:

$$
\begin{array}{ll}
\pi_{1}(a, b)=\frac{2(c-b)}{c^{2}}, & 0<a<1,0<b<c \\
\pi_{2}(a, b)=\frac{1}{c}, & 0<a<1,0<b<c \\
\pi_{3}(a, b)=\frac{2 b}{c^{2}}, & 0<a<1,0<b<c
\end{array}
$$

We can obtained the E-Bayesian estimate of $\theta$ relative to SELF based on $\pi_{1}(a, b)$ which is denoted as $\hat{\theta}_{E B S 1}$ by using (2-7) and (34) in (3-3) to be

$$
\begin{aligned}
\hat{\theta}_{E B S 1} & =\int_{0}^{1} \int_{0}^{c}\left(\frac{r+a}{Q+b}\right)\left[\frac{2(c-b)}{c^{2}}\right] d b d a \\
& =\left(\frac{2 r+1}{c}\right)\left[\left(1+\frac{Q}{c}\right) \ln \left(1+\frac{c}{Q}\right)-1\right]
\end{aligned}
$$

Similarly, we can derive the E-Bayesian estimates of $\theta$ relative to SELF based on $\pi_{2}(a, b)$ and $\pi_{3}(a, b)$ which are denoted as $\hat{\theta}_{E B S}, \hat{\theta}_{E B S 3}$ by using (2-7), (3-5) in (3-3) and (2-7), (3-6) in (3-3) respectively to be

$$
\hat{\theta}_{E B S 2}=\int_{0}^{1} \int_{0}^{c}\left(\frac{r+a}{Q+b}\right)\left[\frac{1}{c}\right] d b d a=\left(\frac{2 r+1}{2 c}\right)\left[\ln \left(1+\frac{c}{Q}\right)\right]
$$

And

$$
\hat{\theta}_{E B S 3}=\int_{0}^{1} \int_{0}^{c}\left(\frac{r+a}{Q+b}\right)\left[\frac{2 b}{c^{2}}\right] d b d a=\left(\frac{2 r+1}{c}\right)\left[1-\frac{Q}{c} \ln \left(1+\frac{c}{Q}\right)\right]
$$

The E-Bayes estimates of $h(t)$ relative to SELF denoted as $\hat{h}_{E B S i}(i=1,2,3)$ can be obtained by replacing $\hat{\theta}_{E B S i}(i=1,2,3)$ given in (3-7), (3-8) and (3-9) instead of $\theta$ given in (1-3) to be

$$
\begin{aligned}
& \hat{h}_{E B S 1}=\lambda e^{\lambda t}\left(\frac{2 r+1}{c}\right)\left[\left(1+\frac{Q}{c}\right) \ln \left(1+\frac{c}{Q}\right)-1\right], \\
& \hat{h}_{E B S 2}=\lambda e^{\lambda t}\left(\frac{2 r+1}{2 c}\right)\left[\ln \left(1+\frac{c}{Q}\right)\right]
\end{aligned}
$$

And

$$
\hat{h}_{E B S 3}=\lambda e^{\lambda t}\left(\frac{2 r+1}{c}\right)\left[1-\frac{Q}{c} \ln \left(1+\frac{c}{Q}\right)\right]
$$

\subsection{E-Bayesian estimation under quadratic loss func- tion (QLF)}

We can obtain the E-Bayesian estimate of $\theta$ relative to QLF based on $\pi_{1}(a, b)$ which is denoted as $\hat{\theta}_{E B Q 1}$ by using (2-11) and (34) in (3-3) to be

$$
\begin{aligned}
\hat{\theta}_{E B Q 1} & =\int_{0}^{1} \int_{0}^{c}\left(\frac{r+a-2}{Q+b}\right)\left[\frac{2(c-b)}{c^{2}}\right] d b d a \\
& =\left(\frac{2 r-3}{c}\right)\left[\left(1+\frac{Q}{c}\right) \ln \left(1+\frac{c}{Q}\right)-1\right]
\end{aligned}
$$

Also, we can derive the E-Bayesian estimates of $\theta$ relative to QLF based on $\pi_{2}(a, b)$ and $\pi_{3}(a, b)$ which are denoted as $\hat{\theta}_{E B Q 2}, \hat{\theta}_{E B Q 3}$ by using (2-11), (3-5) in (3-3) and (2-11), (3-6) in (3$3)$ respectively to be

$$
\hat{\theta}_{E B Q 2}=\int_{0}^{1} \int_{0}^{c}\left(\frac{r+a-2}{Q+b}\right)\left[\frac{1}{c}\right] d b d a=\left(\frac{2 r-3}{2 c}\right)\left[\ln \left(1+\frac{c}{Q}\right)\right]
$$

And

$$
\begin{aligned}
\hat{\theta}_{E B Q 3} & =\int_{0}^{1} \int_{0}^{c}\left(\frac{r+a-2}{Q+b}\right)\left[\frac{2 b}{c^{2}}\right] d b d a \\
& =\left(\frac{2 r-3}{c}\right)\left[1-\frac{Q}{c} \ln \left(1+\frac{c}{Q}\right)\right]
\end{aligned}
$$

Similarly, the E-Bayes estimates of $h(t)$ based on QLF denoted as $\hat{h}_{E B Q i}(i=1,2,3)$ can be obtained by replacing $\hat{\theta}_{E B Q i}(i=1,2,3)$ given in (3-13), (3-14) and (3-15) instead of $\theta$ given in (1-3) to be

$$
\begin{aligned}
& \hat{h}_{E B Q 1}=\lambda e^{\lambda t}\left(\frac{2 r-3}{c}\right)\left[\left(1+\frac{Q}{c}\right) \ln \left(1+\frac{c}{Q}\right)-1\right], \\
& \hat{h}_{E B Q 2}=\lambda e^{\lambda t}\left(\frac{2 r-3}{2 c}\right)\left[\ln \left(1+\frac{c}{Q}\right)\right]
\end{aligned}
$$

And

$$
\hat{h}_{E B Q 3}=\lambda e^{\lambda t}\left(\frac{2 r-3}{c}\right)\left[1-\frac{Q}{c} \ln \left(1+\frac{c}{Q}\right)\right]
$$

\subsection{E-Bayesian estimation under entropy loss function (ELF)}

We can get the E-Bayesian estimate of $\theta$ relative to ELF based on $\pi_{1}(a, b)$ which is denoted as $\hat{\theta}_{E B E 1}$ by using (2-15) and (3-4) in (33) to be

$$
\begin{aligned}
\hat{\theta}_{E B E 1} & =\int_{0}^{1} \int_{0}^{c}\left(\frac{r+a-1}{Q+b}\right)\left[\frac{2(c-b)}{c^{2}}\right] d b d a \\
& =\left(\frac{2 r-1}{c}\right)\left[\left(1+\frac{Q}{c}\right) \ln \left(1+\frac{c}{Q}\right)-1\right]
\end{aligned}
$$

Also, we can derive the E-Bayesian estimates of $\theta$ relative to ELF based on $\pi_{2}(a, b)$ and $\pi_{3}(a, b)$ which are denoted as $\hat{\theta}_{E B E 2}, \hat{\theta}_{E B E 3}$ by using (2-15), (3-5) in (3-3) and (2-15), (3-6) in (3-3) respectively to be

$$
\hat{\theta}_{E B E 2}=\int_{0}^{1} \int_{0}^{c}\left(\frac{r+a-1}{Q+b}\right)\left[\frac{1}{c}\right] d b d a=\left(\frac{2 r-1}{2 c}\right)\left[\ln \left(1+\frac{c}{Q}\right)\right]
$$

And 


$$
\begin{aligned}
\hat{\theta}_{E B E 3} & =\int_{0}^{1} \int_{0}^{c}\left(\frac{r+a-1}{Q+b}\right)\left[\frac{2 b}{c^{2}}\right] d b d a \\
& =\left(\frac{2 r-1}{c}\right)\left[1-\frac{Q}{c} \ln \left(1+\frac{c}{Q}\right)\right]
\end{aligned}
$$

Also, the E-Bayes estimates of $h(t)$ relative to ELF denoted as $\hat{h}_{E B E i}(i=1,2,3)$ can be obtained by replacing $\hat{\theta}_{E B E i}(i=1,2,3)$ given in (3-19), (3-20) and (3-21) instead of $\theta$ given in (1-3) to be

$$
\begin{aligned}
& \hat{h}_{E B E 1}=\lambda e^{\lambda t}\left(\frac{2 r-1}{c}\right)\left[\left(1+\frac{Q}{c}\right) \ln \left(1+\frac{c}{Q}\right)-1\right], \\
& \hat{h}_{E B E 2}=\lambda e^{\lambda t}\left(\frac{2 r-1}{2 c}\right)\left[\ln \left(1+\frac{c}{Q}\right)\right]
\end{aligned}
$$

And

$$
\hat{h}_{E B E 3}=\lambda e^{\lambda t}\left(\frac{2 r-1}{c}\right)\left[1-\frac{Q}{c} \ln \left(1+\frac{c}{Q}\right)\right]
$$

\subsection{E-Bayesian estimation under LINEX Loss function (LLF)}

We can get the E-Bayesian estimate of $\theta$ relative to LLF based on $\pi_{1}(a, b)$ which is denoted as $\hat{\theta}_{E B L 1}$ by using (2-19) and (3-4) in (33) to be

$$
\begin{aligned}
\hat{\theta}_{E B L 1}=\int_{0}^{1} \int_{0}^{c}\left(\frac{r+a}{s}\right) \ln \left[1+\frac{s}{Q+b}\right]\left[\frac{2(c-b)}{c^{2}}\right] d b d a \\
=\left(\frac{2 r+1}{2}\right)\left\{\begin{array}{l}
{\left[\left(\frac{-(Q+c)^{2}}{c^{2} s}\right) \ln \left(1+\frac{c}{Q}\right)\right]+} \\
\left.\left.\left[\frac{(Q+s+c)^{2}}{c^{2} s}\right) \ln \left(1+\frac{c}{Q+s}\right)\right]\right\} \\
+\left[\frac{1}{s} \ln \left(1+\frac{s}{Q}\right)\right]-\left[\frac{1}{c}\right]
\end{array}\right]
\end{aligned}
$$

Also, we can derive the E-Bayesian estimates of $\theta$ relative to LLF based on $\pi_{2}(a, b)$ and $\pi_{3}(a, b)$ which are denoted as $\hat{\theta}_{E B L 2}, \hat{\theta}_{E B L 3}$ by using (2-19), (3-5) in (3-3) and (2-19), (3-6) in (3-3) respectively to be

$$
\begin{aligned}
& \hat{\theta}_{E B L 2}=\int_{0}^{1} \int_{0}^{c}\left(\frac{r+a}{s}\right) \ln \left[1+\frac{s}{Q+b}\right]\left[\frac{1}{c}\right] d b d a \\
& \left.\begin{array}{rl}
=\left(\frac{2 r+1}{2 s}\right)\{\{ & \left.\left[\left(\frac{Q+s}{c}\right) \ln \left(1+\frac{c}{Q+s}\right)\right]-\right\} \\
& {\left[\left(\frac{Q}{c}\right) \ln \left(1+\frac{s}{Q}\right)\right]}
\end{array}\right\}
\end{aligned}
$$

And

$$
\hat{\theta}_{E B L 3}=\int_{0}^{1} \int_{0}^{c}\left(\frac{r+a}{s}\right) \ln \left[1+\frac{s}{Q+b}\right]\left[\frac{2 b}{c^{2}}\right] d b d a
$$

$$
\therefore \hat{\theta}_{E B L 3}=\left(\frac{2 r+1}{2}\right)\left\{\begin{array}{l}
{\left[\left(\frac{-(Q+c)^{2}}{c^{2} s}\right) \ln \left(1+\frac{c}{Q+s}\right)\right]+} \\
{\left[\left(\frac{Q^{2}}{c^{2} s}\right) \ln \left(1+\frac{c}{Q}\right)\right]+} \\
{\left[\frac{1}{s} \ln \left(1+\frac{s}{Q+c}\right)\right]+\left[\frac{1}{c}\right]}
\end{array}\right\}
$$

Also, the E-Bayes estimates of $h(t)$ relative to LLF denoted as $\hat{h}_{E B L i}(i=1,2,3)$ can be obtained by replacing $\hat{\theta}_{E B L i}(i=1,2,3)$ given in (3-25), (3-26) and (3-27) instead of $\theta$ given in (1-3) to be

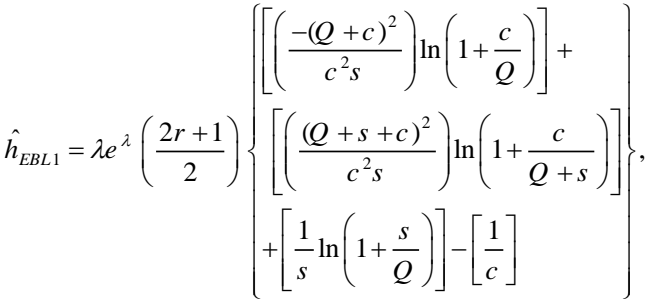

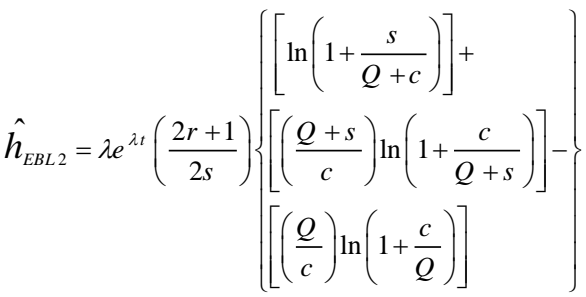

And

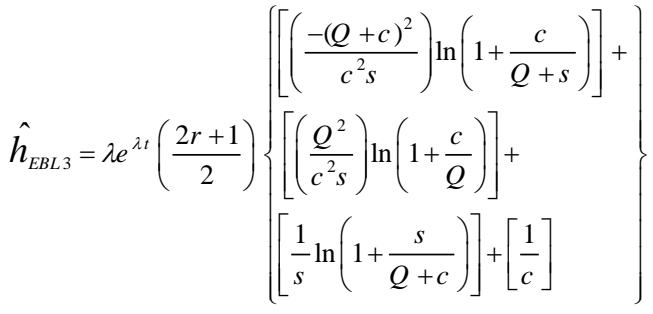

\section{Hierarchical Bayesian estimation}

In this section, we will derive the hierarchical Bayes estimates of the shape parameter $\theta$ and the hazard function $h(t)$ of the Gompertz distribution based on symmetric loss function (SELF)) and three asymmetric loss functions (QLF, ELF and LLF). According to Lindley and Smith [32], if $a$ and $b$ are hyper-parameters in $\theta$, the prior density function of $\theta$ is $g(\theta \mid a, b)$ given in (2-3) and the prior density functions of hyper-parameters $a, b$ are given in (3-4), (3-5) and (3-6), then the corresponding hierarchical prior density functions of $\theta$ are given as the following:

$$
\begin{aligned}
\pi_{4}(\theta) & =\int_{0}^{1} \int_{0}^{c} g(\theta \mid a, b) \pi_{1}(a, b) d b d a \\
& =\frac{2}{c^{2}} \int_{0}^{1} \int_{0}^{c} \frac{b^{a}}{\Gamma(a)}(c-b) \theta^{a-1} e^{-b \theta} d b d a,
\end{aligned}
$$

$$
\begin{aligned}
\pi_{5}(\theta) & =\int_{0}^{1} \int_{0}^{c} g(\theta \mid a, b) \pi_{2}(a, b) d b d a \\
& =\frac{1}{c} \int_{0}^{1} \int_{0}^{c} \frac{b^{a}}{\Gamma(a)} \theta^{a-1} e^{-b \theta} d b d a
\end{aligned}
$$


$\pi_{6}(\theta)=\int_{0}^{1} \int_{0}^{c} g(\theta \mid a, b) \pi_{3}(a, b) d b d a$

$$
=\frac{2}{c^{2}} \int_{0}^{1} \int_{0}^{c} \frac{b^{a+1}}{\Gamma(a)} \theta^{a-1} e^{-b \theta} d b d a
$$

From Bayesian theorem, the hierarchical posterior density functions of $\theta$ can be derived by combining (2-1), (4-1), (4-2) and (43) to be

$$
\begin{aligned}
h_{1}(\theta \mid \underline{x}) & =\frac{L(\theta \mid \underline{x}) \pi_{4}(\theta)}{\int_{0}^{\infty} L(\theta \mid \underline{x}) \pi_{4}(\theta) d \theta} \\
& =\frac{\int_{0}^{1} \int_{0}^{c} \frac{b^{a}}{\Gamma(a)}(c-b) \theta^{r+a-1} e^{-\theta(Q+b)} d b d a}{\int_{0}^{1} \int_{0}^{c} \frac{b^{a}}{\Gamma(a)}(c-b) \Gamma(r+a)[Q+b]^{-(r+a)} d b d a},
\end{aligned}
$$

$$
\begin{aligned}
h_{2}(\theta \mid \underline{x}) & =\frac{L(\theta \mid \underline{x}) \pi_{5}(\theta)}{\int_{0}^{\infty} L(\theta \mid \underline{x}) \pi_{5}(\theta) d \theta} \\
& =\frac{\int_{0}^{1} \int_{0}^{c} \frac{b^{a}}{\Gamma(a)} \theta^{r+a-1} e^{-\theta(Q+b)} d b d a}{\int_{0}^{1} \int_{0}^{c} \frac{b^{a}}{\Gamma(a)} \Gamma(r+a)[Q+b]^{-(r+a)} d b d a},
\end{aligned}
$$

And

$$
\begin{aligned}
h_{3}(\theta \mid \underline{x})= & \frac{L(\theta \mid \underline{x}) \pi_{6}(\theta)}{\int_{0}^{\infty} L(\theta \mid \underline{x}) \pi_{6}(\theta) d \theta} \\
= & \frac{\int_{0}^{1} \int_{0}^{c} \frac{b^{a+1}}{\Gamma(a)} \theta^{r+a-1} e^{-\theta(Q+b)} d b d a}{\int_{0}^{1} \int_{0}^{c} \frac{b^{a+1}}{\Gamma(a)} \Gamma(r+a)[Q+b]^{-(r+a)} d b d a}
\end{aligned}
$$

\subsection{Hierarchical Bayesian estimation under squared error loss function (SELF)}

The hierarchical Bayes estimates of $\theta$ based on SELF denoted by $\hat{\theta}_{H B S i}(i=1,2,3)$ can be obtained as

$\hat{\theta}_{H B S i}=E_{h_{i}}(\theta \mid \underline{x}), \quad i=1,2,3$

Where $E_{h_{i}}$ indicated to the expectation of the hierarchical posterior distribution. We can derived $\hat{\theta}_{H B S i}(i=1,2,3)$ by using (4-4), (45) and (4-6) in (4-7) to be

$$
\begin{gathered}
\hat{\theta}_{H B S 1}=\frac{\int_{0}^{1} \int_{0}^{c} \frac{b^{a}}{\Gamma(a)}(c-b) \Gamma(r+a+1)[Q+b]^{-(r+a+1)} d b d a}{\int_{0}^{1} \int_{0}^{c} \frac{b^{a}}{\Gamma(a)}(c-b) \Gamma(r+a)[Q+b]^{-(r+a)} d b d a}, \\
\hat{\theta}_{H B S 2}=\frac{\int_{0}^{1} \int_{0}^{c} \frac{b^{a}}{\Gamma(a)} \Gamma(r+a+1)[Q+b]^{-(r+a+1)} d b d a}{\int_{0}^{1} \int_{0}^{c} \frac{b^{a}}{\Gamma(a)} \Gamma(r+a)[Q+b]^{-(r+a)} d b d a}
\end{gathered}
$$

And

$$
\hat{\theta}_{H B S 3}=\frac{\int_{0}^{1} \int_{0}^{c} \frac{b^{a+1}}{\Gamma(a)} \Gamma(r+a+1)[Q+b]^{-(r+a+1)} d b d a}{\int_{0}^{1} \int_{0}^{c} \frac{b^{a+1}}{\Gamma(a)} \Gamma(r+a)[Q+b]^{-(r+a)} d b d a}
$$

Similarly, the hierarchical Bayes estimates of $h(t)$ based on SELF denoted as $\hat{h}_{H B S i}(i=1,2,3)$ can be obtained by replacing $\hat{\theta}_{H B S i}(i=1,2,3)$ given in (4-8), (4-9) and (4-10) instead of $\theta$ given in $(1-3)$ to be

$$
\begin{gathered}
\hat{h}_{H B S 1}=\frac{\lambda e^{\lambda t} \int_{0}^{1} \int_{0}^{c} \frac{b^{a}}{\Gamma(a)}(c-b) \Gamma(r+a+1)[Q+b]^{-(r+a+1)} d b d a}{\int_{0}^{1} \int_{0}^{c} \frac{b^{a}}{\Gamma(a)}(c-b) \Gamma(r+a)[Q+b]^{-(r+a)} d b d a}, \\
\hat{h}_{H B S 2}=\frac{\lambda e^{\lambda t} \int_{0}^{1} \int_{0}^{c} \frac{b^{a}}{\Gamma(a)} \Gamma(r+a+1)[Q+b]^{-(r+a+1)} d b d a}{\int_{0}^{1} \int_{0}^{c} \frac{b^{a}}{\Gamma(a)} \Gamma(r+a)[Q+b]^{-(r+a)} d b d a}
\end{gathered}
$$

And

$$
\hat{h}_{H B S 3}=\frac{\lambda e^{\lambda t} \int_{0}^{1} \int_{0}^{c} \frac{b^{a+1}}{\Gamma(a)} \Gamma(r+a+1)[Q+b]^{-(r+a+1)} d b d a}{\int_{0}^{1} \int_{0}^{c} \frac{b^{a+1}}{\Gamma(a)} \Gamma(r+a)[Q+b]^{-(r+a)} d b d a}
$$

\subsection{Hierarchical Bayesian estimation under quadratic loss function (QLF)}

The hierarchical Bayes estimates of $\theta$ based on QLF denoted by $\hat{\theta}_{H B Q i}(i=1,2,3)$ can be obtained as

$\hat{\theta}_{H B Q i}=\frac{E_{h_{i}}\left(\theta^{-1} \mid \underline{x}\right)}{E_{h_{i}}\left(\theta^{-2} \mid \underline{x}\right)} \quad i=1,2,3$

We can derived $\hat{\theta}_{H B Q i}(i=1,2,3)$ by using (4-4), (4-5) and (4-6) in (4-14) to be

$$
\begin{gathered}
\hat{\theta}_{H B Q 1}=\frac{\int_{0}^{1} \int_{0}^{c} \frac{b^{a}}{\Gamma(a)}(c-b) \Gamma(r+a-1)[Q+b]^{-(r+a-1)} d b d a}{\int_{0}^{1} \int_{0}^{c} \frac{b^{a}}{\Gamma(a)}(c-b) \Gamma(r+a-2)[Q+b]^{-(r+a-2)} d b d a}, \\
\hat{\theta}_{H B Q 2}=\frac{\int_{0}^{1} \int_{0}^{c} \frac{b^{a}}{\Gamma(a)} \Gamma(r+a-1)[Q+b]^{-(r+a-1)} d b d a}{\int_{0}^{1} \int_{0}^{c} \frac{b^{a}}{\Gamma(a)} \Gamma(r+a-2)[Q+b]^{-(r+a-2)} d b d a}
\end{gathered}
$$

And

$\hat{\theta}_{H B Q 3}=\frac{\int_{0}^{1} \int_{0}^{c} \frac{b^{a+1}}{\Gamma(a)} \Gamma(r+a-1)[Q+b]^{-(r+a-1)} d b d a}{\int_{0}^{1} \int_{0}^{c} \frac{b^{a+1}}{\Gamma(a)} \Gamma(r+a-2)[Q+b]^{-(r+a-2)} d b d a}$

Similarly, the hierarchical Bayes estimates of $h(t)$ based on QLF denoted as $\hat{h}_{H B Q i}(i=1,2,3)$ can be obtained by replacing $\hat{\theta}_{H B Q i}(i=1,2,3)$ given in (4-15), (4-16) and (4-17) instead of $\theta$ given in (1-3) to be

$$
\hat{h}_{H B Q 1}=\frac{\lambda e^{\lambda t} \int_{0}^{1} \int_{0}^{c} \frac{b^{a}}{\Gamma(a)}(c-b) \Gamma(r+a-1)[Q+b]^{-(r+a-1)} d b d a}{\int_{0}^{1} \int_{0}^{c} \frac{b^{a}}{\Gamma(a)}(c-b) \Gamma(r+a-2)[Q+b]^{-(r+a-2)} d b d a},
$$




$$
\hat{h}_{H B Q 2}=\frac{\lambda e^{\lambda t} \int_{0}^{1} \int_{0}^{c} \frac{b^{a}}{\Gamma(a)} \Gamma(r+a-1)[Q+b]^{-(r+a-1)} d b d a}{\int_{0}^{1} \int_{0}^{c} \frac{b^{a}}{\Gamma(a)} \Gamma(r+a-2)[Q+b]^{-(r+a-2)} d b d a}
$$

And

$$
\hat{h}_{H B Q 3}=\frac{\lambda e^{\lambda t} \int_{0}^{1} \int_{0}^{c} \frac{b^{a+1}}{\Gamma(a)} \Gamma(r+a-1)[Q+b]^{-(r+a-1)} d b d a}{\int_{0}^{1} \int_{0}^{c} \frac{b^{a+1}}{\Gamma(a)} \Gamma(r+a-2)[Q+b]^{-(r+a-2)} d b d a}
$$

\subsection{Hierarchical Bayesian estimation under entropy loss function (ELF)}

The hierarchical Bayes estimates of $\theta$ based on ELF denoted by $\hat{\theta}_{H B E i}(i=1,2,3)$ can be obtained as

$$
\hat{\theta}_{H B E i}=\left[E_{h_{i}}\left(\theta^{-1} \mid \underline{x}\right)\right]^{-1} \quad i=1,2,3
$$

We can derived $\hat{\theta}_{H B E i}(i=1,2,3)$ by using (4-4), (4-5) and (4-6) in (4-21) to be

$$
\begin{gathered}
\hat{\theta}_{H B E 1}=\frac{\int_{0}^{1} \int_{0}^{c} \frac{b^{a}}{\Gamma(a)}(c-b) \Gamma(r+a)[Q+b]^{-(r+a)} d b d a}{\int_{0}^{1} \int_{0}^{c} \frac{b^{a}}{\Gamma(a)}(c-b) \Gamma(r+a-1)[Q+b]^{-(r+a-1)} d b d a}, \\
\hat{\theta}_{H B E 2}=\frac{\int_{0}^{1} \int_{0}^{c} \frac{b^{a}}{\Gamma(a)} \Gamma(r+a)[Q+b]^{-(r+a)} d b d a}{\int_{0}^{1} \int_{0}^{c} \frac{b^{a}}{\Gamma(a)} \Gamma(r+a-1)[Q+b]^{-(r+a-1)} d b d a}
\end{gathered}
$$

And

$$
\hat{\theta}_{H B E 3}=\frac{\int_{0}^{1} \int_{0}^{c} \frac{b^{a+1}}{\Gamma(a)} \Gamma(r+a)[Q+b]^{-(r+a)} d b d a}{\int_{0}^{1} \int_{0}^{c} \frac{b^{a+1}}{\Gamma(a)} \Gamma(r+a-1)[Q+b]^{-(r+a-1)} d b d a}
$$

Similarly, the hierarchical Bayes estimates of $h(t)$ based on ELF denoted as $\hat{h}_{H B E i}(i=1,2,3)$ can be obtained by replacing $\hat{\theta}_{H B E i}(i=1,2,3)$ given in (4-22), (4-23) and (4-24) instead of $\theta$ given in (1-3) to be

$$
\begin{gathered}
\hat{h}_{H B E 1}=\frac{\lambda e^{\lambda t} \int_{0}^{1} \int_{0}^{c} \frac{b^{a}}{\Gamma(a)}(c-b) \Gamma(r+a)[Q+b]^{-(r+a)} d b d a}{\int_{0}^{1} \int_{0}^{c} \frac{b^{a}}{\Gamma(a)}(c-b) \Gamma(r+a-1)[Q+b]^{-(r+a-1)} d b d a}, \\
\hat{h}_{H B E 2}=\frac{\lambda e^{\lambda t} \int_{0}^{1} \int_{0}^{c} \frac{b^{a}}{\Gamma(a)} \Gamma(r+a)[Q+b]^{-(r+a)} d b d a}{\int_{0}^{1} \int_{0}^{c} \frac{b^{a}}{\Gamma(a)} \Gamma(r+a-1)[Q+b]^{-(r+a-1)} d b d a}
\end{gathered}
$$

And

$$
\hat{h}_{H B E 3}=\frac{\lambda e^{\lambda t} \int_{0}^{1} \int_{0}^{c} \frac{b^{a+1}}{\Gamma(a)} \Gamma(r+a)[Q+b]^{-(r+a)} d b d a}{\int_{0}^{1} \int_{0}^{c} \frac{b^{a+1}}{\Gamma(a)} \Gamma(r+a-1)[Q+b]^{-(r+a-1)} d b d a}
$$

\subsection{Hierarchical Bayesian estimation under LINEX loss function (LLF)}

The hierarchical Bayes estimates of $\theta$ based on LLF denoted by $\hat{\theta}_{H B L i}(i=1,2,3)$ can be obtained as

$$
\hat{\theta}_{H B E i}=\left(\frac{-1}{s}\right) \ln \left[E_{h_{i}}\left(e^{-s \theta} \mid \underline{x}\right)\right] \quad i=1,2,3
$$

We can derived $\hat{\theta}_{H B L i}(i=1,2,3)$ by using (4-4), (4-5) and (4-6) in (4-28) to be

$$
\begin{gathered}
\hat{\theta}_{H B L 1}=\left(\frac{-1}{s}\right) \ln \left[\frac{\int_{0}^{1} \int_{0}^{c} \frac{b^{a}}{\Gamma(a)}(c-b) \Gamma(r+a)[Q+b+s]^{-(r+a)} d b d a}{\int_{0}^{1} \int_{0}^{c} \frac{b^{a}}{\Gamma(a)}(c-b) \Gamma(r+a)[Q+b]^{-(r+a)} d b d a}\right], \\
\hat{\theta}_{H B L 2}=\left(\frac{-1}{s}\right) \ln \left[\frac{\int_{0}^{1} \int_{0}^{c} \frac{b^{a}}{\Gamma(a)} \Gamma(r+a)[Q+b+s]^{-(r+a)} d b d a}{\int_{0}^{1} \int_{0}^{c} \frac{b^{a}}{\Gamma(a)} \Gamma(r+a)[Q+b]^{-(r+a)} d b d a}\right]
\end{gathered}
$$

And

$$
\hat{\theta}_{H B L 3}=\left(\frac{-1}{s}\right) \ln \left[\frac{\int_{0}^{1} \int_{0}^{c} \frac{b^{a+1}}{\Gamma(a)} \Gamma(r+a)[Q+b+s]^{-(r+a)} d b d a}{\int_{0}^{1} \int_{0}^{c} \frac{b^{a+1}}{\Gamma(a)} \Gamma(r+a)[Q+b]^{-(r+a)} d b d a}\right]
$$

Similarly, the hierarchical Bayes estimates of $h(t)$ based on LLF denoted as $\hat{h}_{H B L i}(i=1,2,3)$ can be obtained by replacing $\hat{\theta}_{H B L i}(i=1,2,3)$ given in (4-29), (4-30) and (4-31) instead of $\theta$ given in (1-3) to be

$$
\begin{aligned}
& \hat{h}_{H B L 1}=\left(\frac{-\lambda e^{\lambda t}}{s}\right) \ln \left[\frac{\int_{0}^{1} \int_{0}^{c} \frac{b^{a}}{\Gamma(a)}(c-b) \Gamma(r+a)[Q+b+s]^{-(r+a)} d b d a}{\int_{0}^{1} \int_{0}^{c} \frac{b^{a}}{\Gamma(a)}(c-b) \Gamma(r+a)[Q+b]^{-(r+a)} d b d a}\right], \\
& \hat{h}_{H B L 2}=\left(\frac{-\lambda e^{\lambda t}}{s}\right) \ln \left[\frac{\int_{0}^{1} \int_{0}^{c} \frac{b^{a}}{\Gamma(a)} \Gamma(r+a)[Q+b+s]^{-(r+a)} d b d a}{\int_{0}^{1} \int_{0}^{c} \frac{b^{a}}{\Gamma(a)} \Gamma(r+a)[Q+b]^{-(r+a)} d b d a}\right]
\end{aligned}
$$

And

$$
\hat{h}_{H B L 3}=\left(\frac{-\lambda e^{\lambda t}}{s}\right) \ln \left[\frac{\int_{0}^{1} \int_{0}^{c} \frac{b^{a+1}}{\Gamma(a)} \Gamma(r+a)[Q+b+s]^{-(r+a)} d b d a}{\int_{0}^{1} c_{0}^{c^{a+1}} \frac{b^{a}}{\Gamma(a)} \Gamma(r+a)[Q+b]^{-(r+a)} d b d a}\right]
$$

\section{Empirical Bayesian estimation}

The Bayes approach assumed that the hyper-parameters $a$ and $b$ are known. When $a$ and $b$ are unknown, we may use the empirical Bayes criteria to get its estimates from likelihood function and probability density function of the prior distribution [33].Now, from (2-1) and (2-3), the marginal distribution of $x$ given $a$ and $b$ is obtained as:

$f(x \mid a, b) \propto b^{a}[\Gamma(a)]^{-1} \Gamma(r+a)(Q+b)^{-(r+a)}$

By taking the natural log for (5-1), we get

$\log f(x \mid a, b) \propto a \log b-\log \Gamma(a)+\log \Gamma(r+a)-(r+a) \log (Q+b) \quad(5-2)$ 
By taking the derivative for (5-3) and setting it equal to zero, we obtain

$$
\begin{aligned}
\frac{\partial \log f(x \mid a, b)}{\partial a}= & \log b-\frac{\partial}{\partial a} \log \Gamma(a) \\
& +\frac{\partial}{\partial a} \log \Gamma(r+a)-\log (Q+b)=0 \\
\frac{\partial \log f(x \mid a, b)}{\partial b}= & \frac{a}{b}-\frac{(r+a)}{Q+b}=0
\end{aligned}
$$

By solving (5-3) and (5-4) simultaneously, we can get the maximum likelihood estimators of $a$ and $b$ denoted by $\tilde{a}$ and $\tilde{b}$ to be

$$
\tilde{a}=\log \left[\frac{a Q}{r}\right]-\frac{\partial}{\partial a} \log \Gamma(a)+\frac{\partial}{\partial a} \log \Gamma(r+a)-\log \left[Q\left(1+\frac{a}{r}\right)\right]
$$

$\tilde{b}=\frac{\tilde{a} Q}{r}$

\subsection{Empirical Bayesian estimation under squared error loss function (SELF)}

The empirical Bayes estimates of $\theta$ and $h(t)$ based on SELF denoted as $\hat{\theta}_{e B S}$ and $\hat{h}_{e B S}$ respectively can be obtained by replacing $\tilde{a}$ and $\tilde{b}$ in (5-5), (5-6) instead of $a$ and $b$ in (2-7), (2-8) respectively to be

$$
\hat{\theta}_{e B S}=\frac{r+\tilde{a}}{Q+\tilde{b}}
$$

And

$$
\hat{h}_{e B S}=\lambda\left(\frac{r+\tilde{a}}{Q+\tilde{b}}\right) e^{\lambda t}
$$

\subsection{Empirical Bayesian estimation under quadratic loss function (QLF)}

The empirical Bayes estimates of $\theta$ and $h(t)$ relative to on QLF denoted as $\hat{\theta}_{e B Q}$ and $\hat{h}_{e B Q}$ respectively can be obtained by replacing $\tilde{a}$ and $\tilde{b}$ in (5-5), (5-6) instead of $a$ and $b$ in (2-11), (2-12) respectively to be

$$
\hat{\theta}_{e B Q}=\frac{r+\tilde{a}-2}{Q+\tilde{b}}
$$

And

$$
\hat{h}_{e B Q}=\lambda\left(\frac{r+\tilde{a}-2}{Q+\tilde{b}}\right) e^{\lambda t}
$$

\subsection{Empirical Bayesian estimation under entropy Loss function (ELF)}

The empirical Bayes estimates of $\theta$ and $h(t)$ relative to on ELF denoted as $\hat{\theta}_{e B E}$ and $\hat{h}_{e B E}$ respectively can be obtained by replacing $\tilde{a}$ and $\tilde{b}$ in (5-5), (5-6) instead of $a$ and $b$ in (2-15), (2-16) respectively to be

$$
\hat{\theta}_{e B E}=\frac{r+\tilde{a}-1}{Q+\tilde{b}}
$$

And

$$
\hat{h}_{e B E}=\lambda\left(\frac{r+\tilde{a}-1}{Q+\tilde{b}}\right) e^{\lambda t}
$$

\subsection{Empirical Bayesian estimation under LINEX loss function (LLF)}

The empirical Bayes estimates of $\theta$ and $h(t)$ relative to on LLF denoted as $\hat{\theta}_{e B L}$ and $\hat{h}_{e B L}$ respectively can be obtained by replacing $\tilde{a}$ and $\tilde{b}$ in (5-5), (5-6) instead of $a$ and $b$ in (2-19), (2-20) respectively to be

$\hat{\theta}_{e B L}=\left(\frac{r+\tilde{a}}{s}\right) \ln \left[1+\frac{s}{Q+\tilde{b}}\right]$

And

$\hat{h}_{e B L}=\lambda\left(\frac{r+\tilde{a}}{s}\right) \ln \left[1+\frac{s}{Q+\tilde{b}}\right] e^{\lambda t}$

\section{Monte Carlo simulation}

This section conducted a Monte Carlo simulation study to evaluate the performance of different estimators for the shape parameter and hazard function corresponding to the Gompertz distribution discussed in the preceding sections. The simulation structure consists of five basic steps which are:

Step (1): Set the default values (true values) of $\lambda, s$ and $c$ which are 0.4, 2 and 3 respectively. We considered different censoring schemes (different values of $n, r$ ) to observe their effect on the estimates in small, moderate and large dataset which are

\begin{tabular}{cccc}
\hline & small samples & moderate samples & large samples \\
\hline$n$ & 5,10 & $15,20,25$ & 50,70 \\
$r$ & $2,3,4,5$ & $7,12,13,16,18,22$ & $25,30,32,35$ \\
\hline
\end{tabular}

Step (2): For these cases, we generate $a, b$ from the uniform priors distributions $(0,1)$ and $(0, c)$ respectively given in (3-4), (3-5) and (3-6). For given values of $a$ and $b$, we generate $\theta$ from the gamma prior distribution given in (2-3).

Step (3): For known values of $\lambda$, type-II censored samples are generated from the Gompertz distribution with pdf and cdf given in (1-1) and (1-2) respectively through the adoption of inverse transformation method, by using the formula

$t_{i}=F^{-1}\left(U_{i}\right)=\left(\frac{1}{\lambda}\right) \ln \left[1-\left(\frac{1}{\theta}\right) \ln \left(1-U_{i}\right)\right] ; i=1,2, \ldots n$

Where $U$ is a random variable distributed according to uniform distribution on the period $(0,1)$.

Step (4): Calculate the Bayes, E-Bayes, hierarchical Bayes and empirical Bayes estimates of the unknown shape parameter and the hazard function associated to the Gompertz distribution according to the formulas that have been obtained.

Step (5): We repeated this process 10000 times and compute the Mean Square Error (MSE) for the estimates for different censoring schemes and given values of $c, s, \lambda$ where

$\operatorname{MSE}(\hat{\theta})=\frac{1}{10000} \sum_{i=1}^{10000}\left(\hat{\theta}_{i}-\theta\right)^{2}$

And $\hat{\theta}$ stands for an estimator of $\theta$. The simulation results are displayed in Tables (1-8). 
Table 1: Averaged Values of MSEs for Estimates of the Parameter Based on SELF

\begin{tabular}{|c|c|c|c|c|c|c|}
\hline$n$ & $r$ & $\hat{\theta}_{B S}$ & $\hat{\theta}_{E B S}$ & $\hat{\theta}_{H B S}$ & $\hat{\theta}_{e B S}$ & Best estimator \\
\hline \multirow{6}{*}{5} & \multirow{4}{*}{2} & \multirow{4}{*}{0.1154007} & 0.0923816 & 0.0900734 & \multirow{4}{*}{0.1081736} & \multirow{4}{*}{$\begin{array}{c}\text { Hierarchical } \\
\text { Bayes }\end{array}$} \\
\hline & & & 0.1098492 & 0.1034039 & & \\
\hline & & & 0.1319542 & 0.1187886 & & \\
\hline & & & 0.28303229 & 0.7028934 & & \\
\hline & \multirow[t]{3}{*}{3} & \multirow[t]{2}{*}{0.1246743} & 0.1740581 & 0.6009574 & \multirow[t]{2}{*}{0.2037672} & \multirow[t]{2}{*}{ E-Bayes } \\
\hline & & & 0.1115517 & 0.2547409 & & \\
\hline \multirow{6}{*}{10} & & \multirow{4}{*}{0.1073193} & 0.0963787 & 0.0949064 & \multirow{3}{*}{0.1012551} & \multirow{4}{*}{$\begin{array}{c}\text { Hierarchical } \\
\text { Bayes }\end{array}$} \\
\hline & \multirow[t]{3}{*}{4} & & 0.1057411 & 0.1020377 & & \\
\hline & & & 0.1169024 & 0.1096066 & & \\
\hline & & & 0.1170692 & 0.1247767 & & \\
\hline & \multirow[t]{3}{*}{5} & \multirow[t]{3}{*}{0.1102817} & 0.1122438 & 0.1290224 & \multirow[t]{3}{*}{0.1113921} & \multirow[t]{3}{*}{ Bayes } \\
\hline & & & 0.111367 & 0.1141872 & & \\
\hline \multirow{7}{*}{15} & & & 0.1320453 & 0.1327131 & & \\
\hline & \multirow[t]{3}{*}{7} & \multirow[t]{3}{*}{0.1343433} & 0.1343069 & 0.134495 & \multirow[t]{3}{*}{0.1340809} & \multirow[t]{3}{*}{ E-Bayes } \\
\hline & & & 0.1375919 & 0.135698 & & \\
\hline & & & 0.4895673 & 0.4935076 & & \\
\hline & \multirow[t]{3}{*}{12} & 0.4877208 & 0.4882623 & 0.4933152 & 0.5068884 & E-Bayes \\
\hline & & & 0.4872165 & 0.4916517 & & \\
\hline & & & 0.4390487 & 0.4398919 & & \\
\hline & 13 & 0.4387684 & 0.4388826 & 0.4399477 & 0.4604651 & Bayes \\
\hline & & & 0.4388002 & 0.4395233 & & \\
\hline 20 & & & 0.5270064 & 0.5270607 & & \\
\hline & 16 & 0.5270217 & 0.5270204 & 0.5270596 & 0.5338379 & E-Bayes \\
\hline & & & 0.5270347 & 0.5270582 & & \\
\hline & & & 0.5261164 & 0.5261638 & & \\
\hline & 18 & 0.5261348 & 0.5261334 & 0.5261661 & 0.5331317 & E-Bayes \\
\hline & & & 0.5261509 & 0.5261682 & & \\
\hline 25 & & & 0.5385311 & 0.5385329 & & \\
\hline & 22 & 0.5385323 & 0.5385319 & 0.5385327 & 0.5388161 & E-Bayes \\
\hline & & & 0.5385301 & 0.5385325 & & \\
\hline & & & 0.5323611 & 0.5324108 & & \\
\hline & 25 & 0.5323851 & 0.5323835 & 0.5324138 & 0.539386 & E-Bayes \\
\hline 50 & & & 0.5324059 & 0.5324162 & & \\
\hline 50 & & & 0.5530951 & 0.553102 & & \\
\hline & 30 & 0.5530953 & 0.5530952 & 0.5531014 & 0.5539138 & E-Bayes \\
\hline & & & 0.5530954 & 0.5531009 & & \\
\hline & & & 0.5536152 & 0.5536406 & & \\
\hline & 32 & 0.5536197 & 0.5536186 & 0.5536383 & & E-Bayes \\
\hline & & & 0.5536224 & 0.5536366 & 0.5566048 & \\
\hline 70 & & & 0.5564315 & 0.5564399 & & \\
\hline & 35 & 0.5564317 & 0.5564318 & 0.5564391 & 0.5574312 & E-Bayes \\
\hline & & & 0.556432 & 0.5564385 & & \\
\hline
\end{tabular}

Table 2: Averaged Values of MSEs for Estimates of the Parameter $\theta$ Based on QLF

\begin{tabular}{|c|c|c|c|c|c|c|}
\hline$n$ & $r$ & $\hat{\theta}_{B Q}$ & $\hat{\theta}_{E B Q}$ & $\hat{\theta}_{H B Q}$ & $\hat{\theta}_{e B Q}$ & Best estimator \\
\hline \multirow{7}{*}{5} & \multirow{4}{*}{2} & \multirow{4}{*}{0.42377492} & 0.4238526 & 0.4633921 & \multirow{4}{*}{0.4417558} & \multirow{4}{*}{ E-Bayes } \\
\hline & & & 0.4236345 & 0.4658621 & & \\
\hline & & & 0.4456019 & 0.4678147 & & \\
\hline & & & 0.2202763 & 0.2292603 & & \\
\hline & \multirow[t]{3}{*}{3} & \multirow[t]{3}{*}{0.2398602} & 0.2336599 & 0.2350935 & \multirow[t]{3}{*}{0.2200502} & \multirow[t]{3}{*}{ Empirical } \\
\hline & & & 0.2555785 & 0.2435989 & & \\
\hline & & & 0.2378877 & 0.2367211 & & \\
\hline \multirow{5}{*}{10} & 4 & \multirow[t]{3}{*}{0.2523542} & 0.2501698 & 0.2464958 & \multirow[t]{3}{*}{0.2448517} & \multirow[t]{3}{*}{ Hierarchical } \\
\hline & & & 0.2630071 & 0.2548032 & & \\
\hline & & & 0.1798498 & 0.1791594 & & \\
\hline & \multirow[t]{3}{*}{5} & \multirow[t]{3}{*}{0.1899613} & 0.1884004 & 0.1856097 & \multirow[t]{3}{*}{0.1842346} & \multirow[t]{3}{*}{ Hierarchical } \\
\hline & & & 0.1985524 & 0.1918728 & & \\
\hline \multirow{7}{*}{15} & & & 0.1895637 & 0.1887348 & & \\
\hline & 7 & \multirow[t]{3}{*}{0.1960455} & 0.1953579 & 0.1932251 & \multirow[t]{3}{*}{0.1956803} & \multirow[t]{3}{*}{ Hierarchical } \\
\hline & & & 0.2017024 & 0.1973158 & & \\
\hline & & & 0.4951878 & 0.497656 & & \\
\hline & \multirow[t]{3}{*}{12} & \multirow[t]{3}{*}{0.4940261} & 0.4943824 & 0.4974899 & \multirow[t]{3}{*}{0.5118598} & \multirow[t]{3}{*}{ E-Bayes } \\
\hline & & & 0.4937599 & 0.4962776 & & \\
\hline & & & 0.4503912 & 0.4509263 & & \\
\hline \multirow{5}{*}{20} & 13 & \multirow[t]{3}{*}{0.4503843} & 0.4504469 & 0.4510272 & \multirow[t]{3}{*}{0.4712319} & \multirow[t]{3}{*}{ Bayes } \\
\hline & & & 0.4505636 & 0.4508205 & & \\
\hline & & & 0.5283811 & 0.5284392 & & \\
\hline & \multirow[t]{3}{*}{16} & 0.5283964 & 0.5283947 & 0.5284373 & 0.5344438 & E-Bayes \\
\hline & & & 0.5284085 & 0.5284358 & & \\
\hline & & & 0.5274266 & 0.5274779 & & \\
\hline & 18 & 0.5274446 & 0.5274432 & 0.5274792 & 0.5337475 & E-Bayes \\
\hline & & & 0.5274598 & 0.5274806 & & \\
\hline 25 & & & 0.5385601 & 0.538563 & & Bayes \\
\hline & 22 & 0.5385601 & 0.5385601 & 0.5385628 & 0.5388208 & $=$ \\
\hline & & & 0.5385601 & 0.5385627 & & E-Bayes \\
\hline
\end{tabular}




\begin{tabular}{|c|c|c|c|c|c|c|}
\hline \multirow{6}{*}{50} & \multirow{3}{*}{25} & \multirow[b]{2}{*}{0.5340429} & 0.5340203 & 0.5340734 & \multirow{3}{*}{0.5405435} & \multirow{3}{*}{ E-Bayes } \\
\hline & & & 0.5340413 & 0.5340749 & & \\
\hline & & & 0.5340623 & 0.5340762 & & \\
\hline & & & 0.5531793 & 0.5531863 & & \\
\hline & 30 & 0.5531795 & 0.5531794 & 0.5531857 & 0.5539447 & E-Bayes \\
\hline & & & 0.5531796 & 0.5531852 & & \\
\hline & & & 0.5541335 & 0.5541593 & 05560455 & \\
\hline & 32 & 0.5541373 & 0.5541362 & 0.5541568 & 0.5569455 & E-Bayes \\
\hline 70 & & & 0.5541394 & 0.554155 & & \\
\hline & 35 & 0.5565404 & 0.5565404 & 0.5565479 & 0.5574841 & E-Bayes \\
\hline & & & 0.5565407 & 0.5565473 & & \\
\hline
\end{tabular}

Table 3: Averaged Values of MSEs for Estimates of the Parameter $\theta$ Based on ELF

\begin{tabular}{|c|c|c|c|c|c|c|}
\hline$n$ & $r$ & $\hat{\theta}_{B E}$ & $\hat{\theta}_{E B E}$ & $\hat{\theta}_{H B E}$ & $\hat{\theta}_{e B E}$ & Best estimator \\
\hline \multirow{6}{*}{5} & \multirow{3}{*}{2} & \multirow{3}{*}{0.2447842} & 0.2168677 & 0.2165015 & \multirow{3}{*}{0.2393492} & \multirow{3}{*}{$\begin{array}{c}\text { Hierarchical } \\
\text { Bayes }\end{array}$} \\
\hline & & & 0.2382808 & 0.2338617 & & \\
\hline & & & 0.2613634 & 0.2491744 & & \\
\hline & \multirow{3}{*}{3} & \multirow{3}{*}{0.1381134} & 0.1783073 & 0.286921 & \multirow{3}{*}{0.1489339} & \multirow{3}{*}{ Bayes } \\
\hline & & & 0.1505408 & 0.2454365 & & \\
\hline & & & 0.1464825 & 0.1578187 & & \\
\hline \multirow{5}{*}{10} & \multirow{3}{*}{4} & \multirow{3}{*}{0.1687689} & 0.1543769 & 0.1524457 & \multirow{2}{*}{0.1611572} & \multirow{3}{*}{$\begin{array}{c}\text { Hierarchical } \\
\text { Bayes }\end{array}$} \\
\hline & & & 0.1799419 & 0.1714139 & & \\
\hline & & & 0.1355164 & 0.1373493 & \multirow{3}{*}{0.1361098} & \\
\hline & \multirow[t]{3}{*}{5} & \multirow[t]{2}{*}{0.1392281} & 0.1390392 & 0.1396337 & & \multirow[t]{2}{*}{ E-Bayes } \\
\hline & & & 0.1452053 & 0.1414759 & & \\
\hline \multirow{6}{*}{15} & & \multirow{4}{*}{0.1609936} & 0.1561711 & 0.1558313 & \multirow{4}{*}{0.1607174} & \multirow{4}{*}{$\begin{array}{c}\text { Hierarchical } \\
\text { Bayes }\end{array}$} \\
\hline & \multirow[t]{3}{*}{7} & & 0.1605749 & 0.1592189 & & \\
\hline & & & 0.1657474 & 0.1622656 & & \\
\hline & & & 0.4922695 & 0.4954227 & & \\
\hline & & \multirow{2}{*}{0.4907839} & 0.4904062 & 0.4938076 & & \\
\hline & & & 0.4445716 & 0.4452436 & & \\
\hline & 13 & 0.4444365 & 0.4445235 & 0.4453234 & 0.4658121 & Bayes \\
\hline & & & 0.4445488 & 0.4450136 & & \\
\hline 20 & & & 0.5276915 & 0.5277477 & & \\
\hline & 16 & 0.5277067 & 0.5277054 & 0.5277462 & 0.5341407 & E-Bayes \\
\hline & & & 0.5277195 & 0.5277448 & & \\
\hline & & & 0.5267692 & 0.5268187 & & \\
\hline & 18 & 0.5267877 & 0.5267862 & 0.5268205 & 0.5334395 & E-Bayes \\
\hline 25 & & & 0.5268034 & 0.5268223 & & \\
\hline 25 & & & 0.5385450 & 0.5385482 & & \\
\hline & 22 & 0.5385451 & 0.5385451 & 0.5385477 & 0.5388185 & E-Bayes \\
\hline & & & 0.5385451 & 0.5385476 & & \\
\hline & & & 0.5331892 & 0.5332406 & & \\
\hline & 25 & 0.5332126 & 0.5332109 & 0.5332428 & 0.5399644 & E-Bayes \\
\hline & & & 0.5332327 & 0.5332447 & & \\
\hline 50 & & & 0.5531372 & 0.5531442 & & \\
\hline & 32 & 0.5538784 & 0.5538773 & 0.5538974 & 0.5567751 & E-Bayes \\
\hline & & & 0.5538805 & 0.5538956 & & \\
\hline 10 & & & 0.5564859 & 0.5564943 & & \\
\hline & 35 & 0.556486 & 0.5564861 & 0.5564935 & 0.5574577 & E-Bayes \\
\hline & & & 0.5564863 & 0.5564929 & & \\
\hline
\end{tabular}

Table 4: Averaged Values of MSEs for Estimates of the Parameter $\theta$ Based on LLF

\begin{tabular}{|c|c|c|c|c|c|c|}
\hline$n$ & $r$ & $\hat{\theta}_{B L}$ & $\hat{\theta}_{E B L}$ & $\hat{\theta}_{H B L}$ & $\hat{\theta}_{e B L}$ & Best estimator \\
\hline \multirow{6}{*}{5} & \multirow{4}{*}{2} & \multirow{4}{*}{0.1534217} & 0.1300703 & 0.1274319 & \multirow{4}{*}{0.1468433} & \multirow{4}{*}{$\begin{array}{c}\text { Hierarchical } \\
\text { Bayes }\end{array}$} \\
\hline & & & 0.1486116 & 0.1423646 & & \\
\hline & & & 0.1693468 & 0.1570616 & & \\
\hline & & & 0.1164396 & 0.1409541 & & \\
\hline & \multirow[t]{3}{*}{3} & \multirow[t]{3}{*}{0.0999737} & 0.1047927 & 0.1277961 & \multirow[t]{2}{*}{0.1077884} & \multirow[t]{2}{*}{ Bayes } \\
\hline & & & 0.1047618 & 0.1085985 & & \\
\hline \multirow{5}{*}{10} & & & 0.1162738 & 0.1143817 & \multirow{3}{*}{0.1218346} & \multirow{3}{*}{$\begin{array}{c}\text { Hierarchical } \\
\text { Bayes }\end{array}$} \\
\hline & \multirow{2}{*}{4} & \multirow{2}{*}{0.1283687} & 0.1383037 & 0.1307677 & & \\
\hline & & & 0.1115827 & 0.1128788 & & \\
\hline & \multirow[t]{3}{*}{5} & \multirow[t]{3}{*}{0.1145334} & 0.1144052 & 0.114787 & \multirow[t]{3}{*}{0.1121342} & \multirow[t]{2}{*}{ E-Bayes } \\
\hline & & & 0.1193545 & 0.1163856 & & \\
\hline \multirow{4}{*}{15} & & & 0.2035517 & 0.2019998 & & \multirow{3}{*}{$\begin{array}{c}\text { Hierarchical } \\
\text { Bayes }\end{array}$} \\
\hline & \multirow[t]{2}{*}{7} & \multirow[t]{2}{*}{0.2109233} & 0.2101355 & 0.2071128 & \multirow[t]{2}{*}{0.2108287} & \\
\hline & & & 0.4591252 & 0.2122008 & & \\
\hline & 12 & 1.3658455 & 1.3655501 & 1.3660657 & 1.4051697 & E-Bayes \\
\hline
\end{tabular}




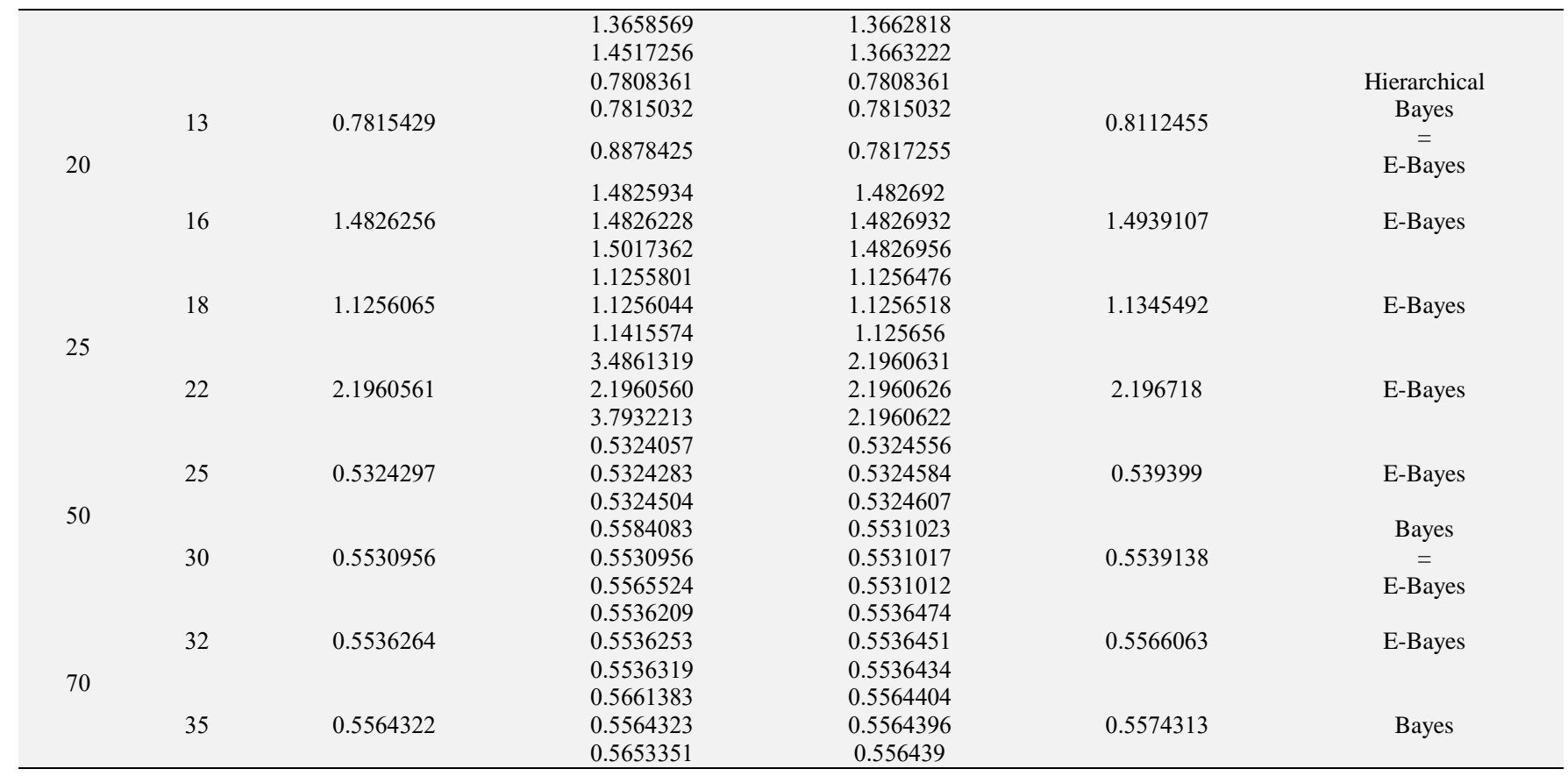

Table 5: Averaged Values of MSEs for Estimates of the Parameter $h(t)$ Based on SELF

\begin{tabular}{|c|c|c|c|c|c|c|}
\hline$n$ & $r$ & $\hat{h}_{B S}$ & $\hat{h}_{E B S}$ & $\hat{h}_{H B S}$ & $\hat{h}_{e B S}$ & Best estimator \\
\hline \multirow{6}{*}{5} & \multirow{3}{*}{2} & \multirow{3}{*}{0.1476429} & 0.1258195 & 0.1227171 & \multirow{3}{*}{0.1412723} & \multirow{3}{*}{$\begin{array}{c}\text { Hierarchical } \\
\text { Bayes }\end{array}$} \\
\hline & & & 0.1426235 & 0.1363939 & & \\
\hline & & & 0.1606808 & 0.1502469 & & \\
\hline & \multirow{4}{*}{3} & \multirow{3}{*}{0.1413497} & 0.120067 & 0.1692736 & \multirow{3}{*}{0.1178139} & \multirow{3}{*}{$\begin{array}{c}\text { Empirical } \\
\text { Bayes }\end{array}$} \\
\hline & & & 0.1343846 & 0.172298 & & \\
\hline & & & 0.1613064 & 0.1511548 & & \\
\hline \multirow{6}{*}{10} & & \multirow{4}{*}{0.1434964} & 0.1313219 & 0.1290326 & \multirow{3}{*}{0.1371602} & \multirow{3}{*}{$\begin{array}{c}\text { Hierarchical } \\
\text { Bayes }\end{array}$} \\
\hline & \multirow[t]{3}{*}{4} & & 0.141665 & 0.1372499 & & \\
\hline & & & 0.1525482 & 0.1455241 & & \\
\hline & & & 0.1565579 & 0.1537777 & \multirow{3}{*}{0.1633304} & \multirow{3}{*}{$\begin{array}{c}\text { Hierarchical } \\
\text { Bayes }\end{array}$} \\
\hline & \multirow[t]{3}{*}{5} & \multirow[t]{2}{*}{0.1701982} & 0.1679724 & 0.1621621 & & \\
\hline & & & 0.1806547 & 0.1717051 & & \\
\hline \multirow{7}{*}{15} & & \multirow{4}{*}{0.1974726} & 0.1896829 & 0.1879971 & \multirow{4}{*}{0.1974171} & \multirow{4}{*}{$\begin{array}{c}\text { Hierarchical } \\
\text { Bayes }\end{array}$} \\
\hline & \multirow[t]{3}{*}{7} & & 0.1965934 & 0.1932869 & & \\
\hline & & & 0.2038466 & 0.198737 & & \\
\hline & & & 1.3653674 & 1.3668662 & & \\
\hline & \multirow[t]{3}{*}{12} & 1.3652581 & 1.3653868 & 1.3670484 & 1.4049949 & Bayes \\
\hline & & & 1.3655327 & 1.3667106 & & \\
\hline & & & 0.7795411 & 0.7796862 & & \\
\hline & 13 & 0.7802063 & 0.7801771 & 0.7801322 & 0.8106249 & E-Bayes \\
\hline 20 & & & 0.7808441 & 0.7804643 & & \\
\hline 20 & & & 1.4825332 & 1.4826317 & & \\
\hline & 16 & 1.4825668 & 1.4825633 & 1.4826333 & 1.4939071 & E-Bayes \\
\hline & & & 1.4825935 & 1.4826361 & & \\
\hline & & & 1.1255281 & 1.1255963 & & \\
\hline & 18 & 1.1255559 & 1.1255537 & 1.1256008 & 1.1345459 & E-Bayes \\
\hline & & & 1.255794 & 1.1256054 & & \\
\hline 25 & & & 2.1960558 & 2.196063 & & \\
\hline & 22 & 2.1960559 & 2.1960559 & 2.1960624 & 2.196718 & E-Bayes \\
\hline & & & 2.1960559 & 2.1960621 & & \\
\hline & & & 0.5719854 & 0.5720236 & & \\
\hline & 25 & 0.572003 & 0.5720018 & 0.5720256 & 0.5770019 & E-Bayes \\
\hline & & & 0.5720182 & 0.5720272 & & \\
\hline 50 & & & 0.7808447 & 0.7808513 & & \\
\hline & 30 & 0.7808449 & 0.7808449 & 0.7808507 & 0.7815812 & E-Bayes \\
\hline & & & 0.780845 & 0.7808503 & & \\
\hline & & & 0.5173308 & 0.5173478 & & \\
\hline & 32 & 0.5173336 & 0.5173329 & 0.5173462 & 0.5192111 & E-Bayes \\
\hline & & & 0.5173351 & 0.517345 & & \\
\hline 70 & & & 0.5868669 & 0.586873 & & \\
\hline & 35 & 0.5868674 & 0.586867 & 0.5868724 & 0.5875585 & E-Bayes \\
\hline & & & 0.5868672 & 0.5868719 & & \\
\hline
\end{tabular}


Table 6: Averaged Values of MSEs for Estimates of the Parameter $h(t)$ Based on QLF

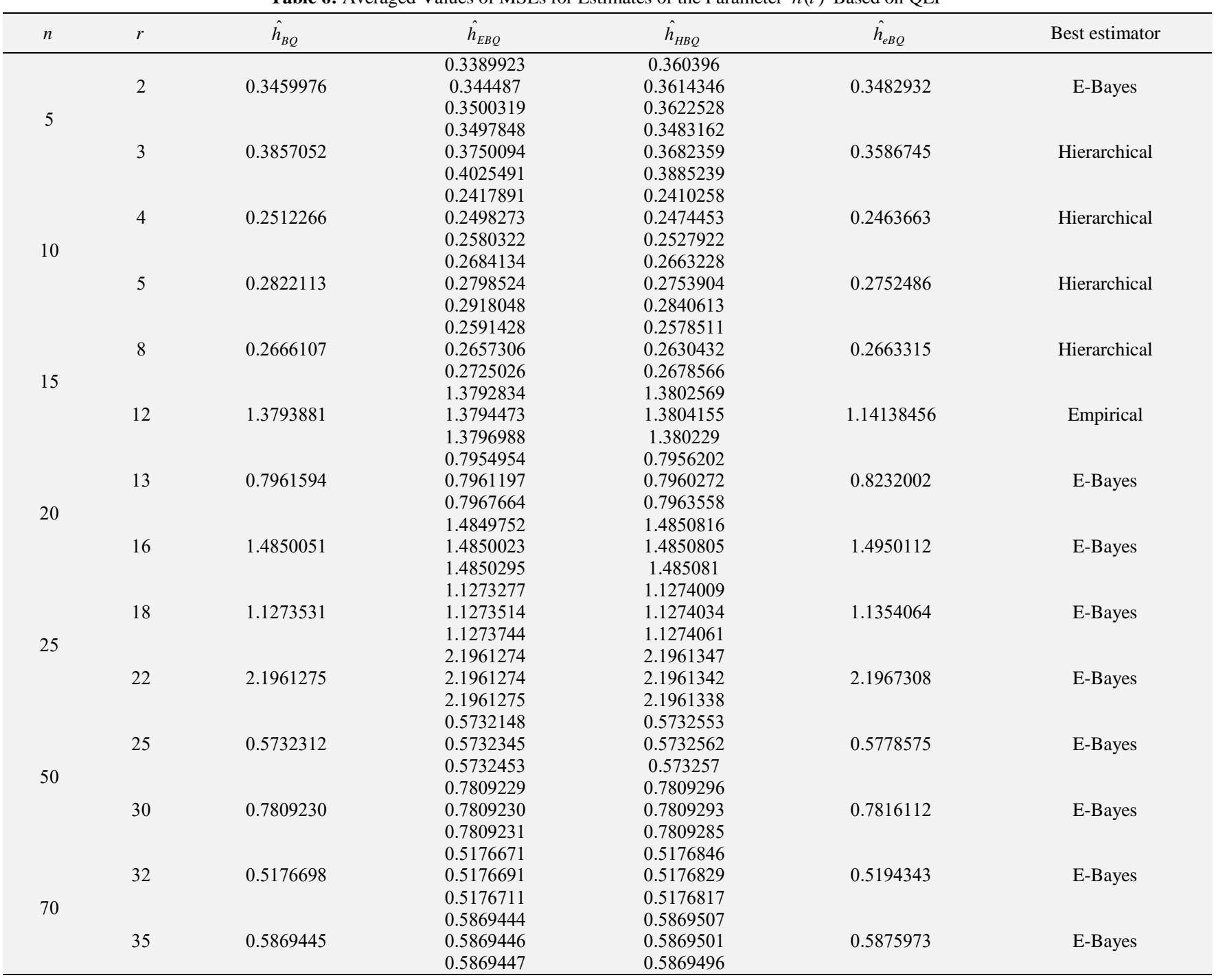

Table 7: Averaged Values of MSEs for Estimates of the Parameter $h(t)$ Based on ELF

\begin{tabular}{|c|c|c|c|c|c|c|}
\hline$n$ & $r$ & $\hat{h}_{B E}$ & $\hat{h}_{E B E}$ & $\hat{h}_{H B E}$ & $\hat{h}_{e B E}$ & Best estimator \\
\hline \multirow{6}{*}{5} & \multirow{3}{*}{2} & \multirow{3}{*}{0.2363194} & 0.2191796 & 0.2189966 & \multirow{3}{*}{0.2331493} & \multirow{3}{*}{$\begin{array}{c}\text { Hierarchical } \\
\text { Bayes }\end{array}$} \\
\hline & & & 0.2324628 & 0.229798 & & \\
\hline & & & 0.2461973 & 0.2389718 & & \\
\hline & \multirow{3}{*}{3} & \multirow{3}{*}{0.2446143} & 0.2070463 & 0.2117466 & \multirow{3}{*}{0.2129794} & \multirow{3}{*}{ E-Bayes } \\
\hline & & & 0.2331799 & 0.2281944 & & \\
\hline & & & 0.2657445 & 0.2453087 & & \\
\hline \multirow{5}{*}{10} & \multirow{3}{*}{4} & \multirow{2}{*}{0.1934148} & 0.1820682 & 0.1804084 & \multirow{2}{*}{0.1875475} & \multirow{2}{*}{$\begin{array}{c}\text { Hierarchical } \\
\text { Bayes }\end{array}$} \\
\hline & & & 0.201693 & 0.195379 & & \\
\hline & & \multirow{3}{*}{0.2216547} & 0.2072122 & 0.2044472 & \multirow{3}{*}{0.2144449} & \multirow{3}{*}{$\begin{array}{c}\text { Hierarchical } \\
\text { Bayes }\end{array}$} \\
\hline & \multirow[t]{2}{*}{5} & & 0.2192354 & 0.2137031 & & \\
\hline & & & 0.2321073 & 0.2234342 & & \\
\hline \multirow{5}{*}{15} & \multirow{4}{*}{7} & \multirow{4}{*}{0.2303126} & 0.222526 & 0.2209862 & \multirow{4}{*}{0.2301584} & \multirow{4}{*}{$\begin{array}{c}\text { Hierarchical } \\
\text { Bayes }\end{array}$} \\
\hline & & & 0.2294133 & 0.2263544 & & \\
\hline & & & 0.236558 & 0.231613 & & \\
\hline & & & 1.3722569 & 1.3734691 & & \\
\hline & \multirow{2}{*}{12} & \multirow{2}{*}{1.3722642} & 1.3725596 & 1.3733802 & \multirow{2}{*}{1.4094141} & \multirow{2}{*}{ E-Bayes } \\
\hline \multirow{6}{*}{20} & & & 0.7874409 & 0.7875702 & & \\
\hline & \multirow[t]{3}{*}{13} & \multirow[t]{3}{*}{0.7881094} & 0.7880746 & 0.7879983 & 0.8168902 & E-Bayes \\
\hline & & & 0.7887349 & 0.7883314 & & \\
\hline & & & 1.4837526 & 1.4838549 & & \\
\hline & 16 & 1.4837841 & 1.4837812 & 1.4838552 & 1.494459 & E-Bayes \\
\hline & & & 1.4838109 & 1.4838568 & & \\
\hline & & & 1.1264267 & 1.1264973 & & \\
\hline & 18 & 1.1264533 & 1.1264512 & 1.1265008 & 1.1349761 & E-Bayes \\
\hline 25 & & & 1.1264757 & 1.1265045 & & \\
\hline 25 & & & 2.1960916 & 2.1960988 & & \\
\hline & 22 & 2.1960917 & 2.1960917 & 2.1960983 & 2.1967244 & E-Bayes \\
\hline & & & 2.1960917 & 2.1960979 & & \\
\hline
\end{tabular}




\begin{tabular}{|c|c|c|c|c|c|c|}
\hline \multirow{6}{*}{50} & \multirow{3}{*}{25} & \multirow[b]{2}{*}{0.5726165} & 0.5725995 & 0.5726388 & \multirow{3}{*}{0.5774295} & \multirow[b]{2}{*}{ E-Bayes } \\
\hline & & & 0.5726153 & 0.5726403 & & \\
\hline & & & 0.5726312 & 0.5726415 & & \\
\hline & \multirow{4}{*}{30} & \multirow{4}{*}{0.7808846} & 0.7808838 & 0.7808904 & \multirow{4}{*}{0.7815962} & \multirow{4}{*}{ E-Bayes } \\
\hline & & & 0.7808839 & 0.7808899 & & \\
\hline & & & 0.7808841 & 0.7808894 & & \\
\hline \multirow{5}{*}{70} & & & 0.5174989 & 0.5175161 & & \\
\hline & \multirow[t]{2}{*}{32} & \multirow[t]{2}{*}{0.5175017} & 0.517501 & 0.5175145 & \multirow[t]{2}{*}{0.5193227} & \multirow[t]{2}{*}{ E-Bayes } \\
\hline & & & 0.517503 & 0.5175133 & & \\
\hline & \multirow[t]{2}{*}{35} & \multirow[t]{2}{*}{0.5869058} & 0.5869058 & 0.5869112 & \multirow[t]{2}{*}{0.5875779} & \multirow[t]{2}{*}{ E-Bayes } \\
\hline & & & 0.586906 & 0.5869108 & & \\
\hline
\end{tabular}

Table 8: Averaged Values of MSEs for Estimates of the Parameter $h(t)$ Based on LLF

\begin{tabular}{|c|c|c|c|c|c|c|}
\hline$n$ & $r$ & $\hat{h}_{B L}$ & $\hat{h}_{E B L}$ & $\hat{h}_{H B L}$ & $\hat{h}_{e B L}$ & Best estimator \\
\hline \multirow{7}{*}{5} & \multirow{4}{*}{2} & \multirow{4}{*}{0.1763238} & 0.1592111 & 0.1571097 & \multirow{4}{*}{0.1716604} & \multirow{4}{*}{ Hierarchical } \\
\hline & & & 0.1729069 & 0.1683651 & & \\
\hline & & & 0.2923221 & 0.1788406 & & \\
\hline & & & 0.1585437 & 0.1523755 & & \\
\hline & \multirow[t]{3}{*}{3} & \multirow[t]{3}{*}{0.1897742} & 0.1820938 & 0.1690574 & \multirow[t]{3}{*}{0.1628875} & \multirow[t]{3}{*}{ Hierarchical } \\
\hline & & & 0.4584379 & 0.1903974 & & \\
\hline & & & 0.1519278 & 0.1500465 & & \\
\hline \multirow{5}{*}{10} & 4 & \multirow[t]{3}{*}{0.1626394} & 0.161177 & 0.1577888 & \multirow[t]{3}{*}{0.1570101} & \multirow[t]{3}{*}{ Hierarchical } \\
\hline & & & 0.3430544 & 0.1645925 & & \\
\hline & & & 0.1799092 & 0.1771111 & & \\
\hline & \multirow[t]{3}{*}{5} & \multirow[t]{3}{*}{0.1929771} & 0.1910202 & 0.1856013 & \multirow[t]{3}{*}{0.1864011} & \multirow[t]{3}{*}{ Hierarchical } \\
\hline & & & 0.4555219 & 0.1946963 & & \\
\hline \multirow{7}{*}{15} & & & 0.2035517 & 0.2019998 & & \\
\hline & \multirow[t]{3}{*}{7} & \multirow[t]{3}{*}{0.2109233} & 0.2101355 & 0.2071128 & \multirow[t]{3}{*}{0.2108287} & \multirow[t]{3}{*}{ Hierarchical } \\
\hline & & & 0.4591252 & 0.2122008 & & \\
\hline & & & 1.3655501 & 1.3660657 & & \\
\hline & \multirow[t]{3}{*}{12} & \multirow[t]{3}{*}{1.3658455} & 1.3658569 & 1.3662818 & 1.4051697 & E-Bayes \\
\hline & & & 1.4517256 & 1.3663222 & & \\
\hline & & & 0.7808360 & 0.7808360 & & Hierarchical \\
\hline & 13 & 0.7815429 & 0.7815032 & 0.7815032 & 0.8112455 & $=$ \\
\hline & & & 0.8878343 & 0.7817255 & & E-Bayes \\
\hline 20 & & & 1.4825934 & 1.482692 & & \\
\hline & 16 & 1.4826256 & 1.4826228 & 1.4826932 & 1.4939107 & E-Bayes \\
\hline & & & 1.5017362 & 1.4826956 & & \\
\hline & & & 1.1255801 & 1.1256476 & & \\
\hline & 18 & 1.1256065 & 1.1256044 & 1.1256518 & 1.1345492 & E-Bayes \\
\hline & & & 1.1415574 & 1.125656 & & \\
\hline 25 & & & 3.4861319 & 2.1960631 & & \\
\hline & 22 & 2.1960561 & 2.1960560 & 2.1960626 & 2.196718 & E-Bayes \\
\hline & & & 3.7932213 & 2.1960622 & & \\
\hline & & & 0.5720181 & 0.5720563 & & \\
\hline & 25 & 0.5720357 & 0.5720345 & 0.5720583 & 0.5770112 & E-Bayes \\
\hline 50 & & & 0.5773542 & 0.5720598 & & \\
\hline 50 & & & 0.7873262 & 0.7808516 & & Bayes \\
\hline & 30 & 0.7808452 & 0.7808452 & 0.7808513 & 0.7815812 & $=$ \\
\hline & & & 0.7861364 & 0.7808506 & & E-Bayes \\
\hline & & & 0.5173338 & 0.517352 & & \\
\hline & 32 & 0.5173379 & 0.5173372 & 0.5173504 & 0.5192121 & E-Bayes \\
\hline & & & 0.5226816 & 0.5173493 & & \\
\hline 70 & & & 0.5954512 & 0.5868733 & & Bayes \\
\hline & 35 & 0.5868673 & 0.5868673 & 0.5868727 & 0.5875585 & $=$ \\
\hline & & & 0.5961556 & 0.5868723 & & E-Bayes \\
\hline
\end{tabular}

\section{Conclusion remarks}

- Among four estimates of $\theta$ based on SELF shown in Table 1 , we can deducted that hierarchical Bayes estimates are the best estimators in most cases of small samples sizes [5], [2], [10], [4], while the E-Bayes are the best estimators in [5], [3] and the Bayes estimators are the best in [10], [5].Also, the E-Bayes estimates have smallest MSE in nearly all cases of moderate and large sample sizes except for [20], [13] where the Bayes estimates are the best. Generally, the overall performance of the four techniques for estimating $\theta$ can be ordered due to number of having smaller MSE as follows:
- Among four estimates of $\theta$ based on QLF shown in Table 2 , we can deducted that hierarchical Bayes estimates are the best estimators in most cases of small samples sizes [10], [4], [10], [5], while the E-Bayes are the best estimators in [5], [2] and the empirical Bayes estimators are the best in [5], [3]. In addition, the E-Bayes estimates have smallest MSE in most cases of moderate sample sizes except for [15], [7] where the hierarchical Bayes estimates are the best, [20], [13] where the Bayes estimates are the best and [25], [22] where the Bayes and E-Bayes estimates are equivalent. In large samples, the E-Bayes estimates are the best in all cases. Generally, the overall performance of the four methods for estimating $\theta$ can be ordered due to number of having smaller MSE as follows:

$$
\hat{\theta}_{E B Q}>\hat{\theta}_{H B Q}>\hat{\theta}_{B Q}>\hat{\theta}_{e B Q}
$$

$$
\hat{\theta}_{E B S}>\hat{\theta}_{B S}=\hat{\theta}_{H B S}>\hat{\theta}_{e B S}
$$


- Among four estimates of $\theta$ based on ELF shown in Table 3 , we can deducted that hierarchical Bayes estimates have smallest MSE in most cases of small samples sizes [5], [2], [10], [4], while the Bayes are the best estimators in [5], [3] and the E-Bayes estimators are the best in [10], [5]. Furthermore, the E-Bayes estimates are the best in most cases of moderate sample sizes except for [15], [7] where the hierarchical Bayes estimates are the best and [20], [13] where the Bayes estimates are the best. In large samples, the EBayes have smallest MSE in nearly all cases except for [50], [30] where the empirical Bayes estimates are the best. Generally, the overall performance of the four methods for estimating $\theta$ can be ordered due to number of having smaller MSE as follows:

$$
\hat{\theta}_{E B E}>\hat{\theta}_{H B E}>\hat{\theta}_{B E}>\hat{\theta}_{e B E}
$$

- Among four estimates of $\theta$ based on LLF shown in Table 4 , we can deducted that hierarchical Bayes estimates have smallest MSE in most cases of small samples sizes [5], [2], [10], [4], while the Bayes estimates have smallest MSE in [5], [3] and the E-Bayes estimators are the best in [10], [5]. Also, the E-Bayes estimates are the best in most cases of moderate sample sizes except for [15], [7] where the hierarchical Bayes estimates are the best and [20], [13] where the E-Bayes estimates and hierarchical Bayes estimates are equivalent. In large samples, the E-Bayes have smallest MSE in nearly all cases except for [50], [30] where the EBayes and Bayes estimates are equivalent. Generally, the overall performance of the four methods for estimating $\theta$ can be ordered due to number of having smaller MSE as follows:

$$
\hat{\theta}_{E B L}>\hat{\theta}_{H B L}>\hat{\theta}_{B L}>\hat{\theta}_{e B L}
$$

- In comparing the various techniques relative to the different loss functions in estimating $\theta$, we can ordered them due to having smallest MSE to be

$$
\hat{\theta}_{S E L F}>\hat{\theta}_{L L F}>\hat{\theta}_{E L F}>\hat{\theta}_{Q L F}
$$

- Among four estimates of $h(t)$ based on SELF shown in Table 5, we can deducted that hierarchical Bayes estimates have smallest MSE in nearly all cases of small samples sizes except for [5], [3], while the empirical Bayes estimates are the best. In addition, the E-Bayes estimates are the best in most cases of moderate sample sizes except for [15], [7] where the hierarchical Bayes estimates are the best and [15], [12] where the Bayes estimates are the best. In large samples, the E-Bayes have smallest MSE in all cases. Generally, the overall performance of the four methods for estimating $h(t)$ can be ordered due to number of having smaller MSE as follows:

$$
\hat{h}_{E B S}>\hat{h}_{H B S}>\hat{h}_{B S}=\hat{h}_{e B S}
$$

- Among four estimates of $h(t)$ based on QLF shown in Table 6 , we can deducted that hierarchical Bayes estimates have smallest MSE in nearly all cases of small samples sizes except for [5], [2] where the E-Bayes estimates are the best. Also, the E-Bayes estimates are the best in most cases of moderate sample sizes except for [15], [7] where the hierarchical Bayes estimates are the best and [15], [12] where the empirical Bayes estimates are the best. In large samples, the E-Bayes have smallest MSE in all cases. Generally, the overall performance of the four methods for estimating $h(t)$ can be ordered due to number of having smaller MSE as follows:

$$
\hat{h}_{E B Q}>\hat{h}_{H B Q}>\hat{h}_{e B Q}>\hat{h}_{B Q}
$$

- Among four estimates of $h(t)$ based on ELF shown in Table 7, we can deducted that hierarchical Bayes estimates are the best in nearly all cases of small samples sizes except for [5], [3] where the E-Bayes estimates are the best. Furthermore, the E-Bayes estimates are the best in nearly all cases of moderate and large sample sizes except for [15], [7] where the hierarchical Bayes estimates are the best. Generally, the overall performance of the four methods for estimating $h(t)$ can be ordered due to number of having smaller MSE as follows:

$$
\hat{h}_{E B E}>\hat{h}_{H B E}>\hat{h}_{B E}=\hat{h}_{e B E}
$$

- Among four estimates of $h(t)$ based on LLF shown in Table 8 , we can deducted that hierarchical Bayes estimates are the best in all cases of small samples sizes. Also, the EBayes estimates are the best in nearly all cases of moderate samples sizes except for [15], [7] where the hierarchical Bayes estimates are the best and [20], [13] where the EBayes and hierarchical Bayes estimates are equivalent. In the end, The E-Bayes estimates have smallest MSE in most cases except for [50], [30], [70], [35] where the Bayes and E-Bayes and Bayes estimates are equivalent. Generally, the overall performance of the four methods for estimating $h(t)$ can be ordered due to number of having smaller MSE as follows:

$$
\hat{h}_{E B L}>\hat{h}_{H B L}>\hat{h}_{B L}=\hat{h}_{e B L}
$$

- In comparing the different approaches within the various loss function, we can ordered them due to having smallest MSE to be

$$
\hat{h}_{S E L F}>\hat{h}_{L L F}>\hat{h}_{E L F}>\hat{h}_{Q L F}
$$

\section{References}

[1] B. Gompertz, on the nature of the function expressive of the law of human mortality and on the new mode of determining the value of life contingencies, Philosophical Transactions of Royal Society A, 115 (1825) 513-580. http://dx.doi.org/10.1098/rstl.1825.0026

[2] M.L. Grag, B.R. Rao, C.K. Redmond, Maximum likelihood estimation of the parameters of the Gompertz survival function, Journal of Royal Statistics Society,19 (1970)152-159. http://dx.doi.org/10.2307/2346545.

[3] Z. Chen, Parameter estimation of the Gompertz population, Bio$\begin{array}{lll}\text { metrical Journal, 39(1997)117-124. } & \end{array}$ http://dx.doi.org/10.1002/bimj.4710390111.

[4] Z. F. Jaheen, A Bayesian analysis of record statistics from Gompertz model, Applied Mathematical Computations, 145(2003) 307 320. http://dx.doi.org/10.1016/S0096-3003(02)00489-7.

[5] S. J. Wu, C. T. Chang, T. R. Tsai, Point and interval estimators for the Gompertz distribution under progressive type-II censoring, Merton International Journal of Statistics, 21, 3(2003)403-418.

[6] A. Khedhairi, A. E. Gohary, A new class of bivariate Gompertz distributions and its mixture, International Journal of Mathematical Analysis, 2, 5(2008) 235-253

[7] B. Saracoglu, M.F. Kaya, A. M. Abd-Elfattah, Comparisons of estimators for stress-strength reliability in Gompertz case, Hacettepe Journal of Mathematics and Statistics, 38, 3(2009) 339-349.

[8] A. A. Ismail, Planning step-sress life tests with type-II censored data, Scientific Research and Essays, 6,19(2011)4021-4028.

[9] N. Feroze, M. Aslam, On Bayesian estimation and predictions for two-components mixture of the Gompertz distribution, Journal of Modern Applied Statistics, 12, 2(2013)269-292. 
[10] J. M. Sarabia, E. Gomez-Deniz, P. Faustino, J. Vanesa, Explicit expressions for premiums and risk measures for the Gompertz distribution, IBIT-XV Iberian-Italian Congress of Financial and Actuarial Mathematics Steville,23-24(2014) 1-24.

[11] M. Han, Expected Bayesian Method for Forecast of Security Investment, Journal of Operations Research and Management Science 14, 5 (2005) 89-102.

[12] M. Han, E-Bayesian Method to Estimate Failure Rate, The Sixth International Symposium on Operations Research and Its Applications (ISOR06) Xinjiang (2006)299-311.

[13] M. Han, E-Bayesian estimation of failure probability and its applications, Journal of Mathematics and Computer Modelling 45 (2007) 1272-1279. http://dx.doi.org/10.1016/j.mcm.2006.11.007.

[14] Q. Yin, H. Liu, Bayesian estimation of geometric distribution parameter under scaled squared error loss function, Conference on Environmental Science and Information Application Technology (2010)650-653.

[15] M. Han, E-Bayesian estimation of the reliability derived from Binomial distribution, Journal of Applied Mathematical Modelling 35 (2011) 2419-2424. http://dx.doi.org/10.1016/j.apm.2010.11.051.

[16] J. Wei, B. Song, W. Yan, Z. Mao, Reliability Estimations of BurrXII Distribution under Entropy Loss Function, IEEE (2011) 244 247. http://dx.doi.org/10.1109/icrms.2011.5979276.

[17] Z. F. Jaheen, H. M. Okasha, E-Bayesian Estimation for the Burr type XII model based on type-2 censoring, Applied Mathematical $\begin{array}{lllll}\text { Modelling } & 35 & \text { (2011) } & 4730 & -\end{array}$ http://dx.doi.org/10.1016/j.apm.2011.03.055.

[18] G. Cai, W. Xu, W. Zhang, P. Wang, Application of E-Bayes method in stock forecast, Fourth International Conference on Information and Computing (2011)504-506 http://dx.doi.org/10.1109/icic.2011.40.

[19] H. M. Okasha, E-Bayesian estimation of system reliability with Weibull distribution of components based on type- 2 censoring, Journal of Advanced Research in Scientific Computing 4,4 (2012)34-45.

[20] X. Wu, E-Bayesian Estimation of Failure Probability under Zerofailure Data with Double Hyper Parameters, Journal of Applied Mechanics and Materials 190-191 (2012) 977-981. http://dx.doi.org/10.4028/www.scientific.net/AMM.190-191.977.

[21] R. Azimi, F, Yaghamei, B. Fasihi, E-Bayesian estimation based on generalized half Logistic progressive type-II censored data, International Journal of Advanced Mathematical Science 1, 2 (2013) 56-63.

[22] N. Javadkani, P. Azhdari, R. Azimi, On Bayesian estimation from two parameter Bathtub-shaped lifetime distribution based on progressive first-failure-censored sampling, International Journal of Scientific World 2, 1 (2014) 31-41. http://dx.doi.org/10.14419/ijsw.v2i1.2513.

[23] H. Liu, T. Yin, C. Wang, E-Bayes Estimation of Rayleigh Distribution Parameter, Journal of Applied Mechanics and Materials 596 (2014) 305-308. http://dx.doi.org/10.4028/www.scientific.net/AMM.596.305.

[24] H. M. Okasha, E-Bayesian Estimation for the Lomax distribution based on type-II censored data, Journal of the Egyptian Mathematical Society 22, 3 (2014) 489-495. http://dx.doi.org/10.1016/j.joems.2013.12.009.

[25] H. M. Reyad, S. O. Ahmed, E-Bayesian analysis of the Gumbel type-ii distribution under type-ii censored scheme, International Journal of Advanced Mathematical Sciences 3, 2 (2015) 108-120. http://dx.doi.org/10.14419/ijams.v3i2.5093.

[26] A. Mood, F. A. Graybill, D. Boes, Introduction to the Theory of Statistics. McGraw-Hill Series in Probability and Statistics, 1974.

[27] M. K. Bhuiyan, M. K. Roy, M. F. Iman, Minimax estimation of the parameter of Rayleigh distribution, (2007) 207-212.

[28] D. K. Dey, M. Gosh, C. Srinivasan, Simultaneous estimation of parameter under entropy loss, Journal of Statistical Planning and Inference (1987) 347-363.

[29] A. Zellner, Bayesian estimation and Prediction using Asymmetric loss Function. Journal of American Statistical Association 81 (1986) 446-451. http://dx.doi.org/10.1080/01621459.1986.10478289.

[30] M. Han, The structure of hierarchical prior distribution and its applications, Chinese Operations Research and Management Science 6,3 (1997) 31-40.

[31] M. Han, E- Bayesian estimation and hierarchical Bayesian estimation of failure rate, Applied Mathematical Modelling 33 (2009) 1915-1922. http://dx.doi.org/10.1016/j.apm.2008.03.019.

[32] D. V. Lindley, A. F. Smith, Bayes estimation for the linear model, Journal of Royal Statistical Society B, 34 (1972)1-41.

[33] O. Shojaee, R. Azimi, M. Babanezhad, Empirical Bayes Estimators of Parameter and Reliability Function for Compound Rayleigh Distribution under Record Data, American Journal of Theoretical and
Applied Statistics $\quad 1$ (2012) 